Pacific Journal of Mathematics

WEAK COMPACTNESS IN SPACES OF COMPACT
OPERATORS AND OF VECTOR-VALUED FUNCTIONS 


\title{
WEAK COMPACTNESS IN SPACES OF COMPACT OPERATORS AND OF VECTOR-VALUED FUNCTIONS
}

\author{
Heron S. Collins and Wolfgang Ruess
}

\begin{abstract}
This is a study of weak compactness and weak convergence in spaces of compact operators and in spaces of vector-valued functions. The link between these two kinds of spaces is provided by the $\varepsilon$-product $X_{\varepsilon} Y$ of locally convex spaces $X$ and $Y$ as introduced by Laurent Schwartz. Spaces of compact operators, like $K(X, Y)$ and $X \tilde{\otimes}_{\varepsilon} Y$, and spaces of vector-valued functions, like $C(K, X)$, and many more concrete spaces of analysis can be represented as (linear subspaces of) suitable $\varepsilon$-products. Accordingly, the program of this paper is to characterize (i) weak compactness, (ii) weak conditional compactness, (iii) weak sequential convergence, and (iv) reflexivity in the general context of $X \varepsilon Y$, and then to specialize the results to (a) spaces of compact operators, (b) injective tensor products, and (c) spaces of vector-valued continuous, or Pettis integrable functions.
\end{abstract}

0.1 Introduction. In this paper we study various concepts of weak compactness and weak convergence in the $\varepsilon$-product $X \varepsilon Y$ of locally convex spaces $X$ and $Y$, and specialize the results to the many operator and function spaces of analysis which can be represented as topological linear subspaces of suitable $\varepsilon$-products: (a) spaces of compact linear operators, (b) injective tensor products, and (c) spaces of vector-valued continuous, holomorphic, or Pettis integrable functions.

The $\varepsilon$-product $X \varepsilon Y$ of locally convex spaces $X$ and $Y$ is the operator space $L_{e}\left(X_{c}^{\prime}, Y\right)$ of all weak*-weakly continuous linear operators from $X^{\prime}$ into $Y$ which transform equicontinuous subsets of $X^{\prime}$ into relatively compact subsets of $Y$, endowed with the topology of uniform convergence on the equicontinuous sets in $X^{\prime}$. For Banach spaces $X$ and $Y$, this is the familiar space of compact weak*-weakly continuous linear operators from $X^{\prime}$ into $Y$, with the operator norm.

The main object of our considerations is to characterize the various concepts of weak convergence in the operator space $L_{e}\left(X_{c}^{\prime}, Y\right)$ in terms of the corresponding concepts in the (presumably well-known) factor spaces $X$ and $Y$.

We now briefly describe the contents of the paper.

Section 1 contains the fundamental results on weak [conditional] compactness in $L_{e}\left(X_{c}^{\prime}, Y\right)$, together with applications to spaces of compact operators and to injective tensor products. 
Section 2 deals with the special problem of weak convergence for sequences in $L_{e}\left(X_{c}^{\prime}, Y\right)$. We determine the form of the extreme points in the dual of $L_{e}\left(X_{c}^{\prime}, Y\right)$ (and in the duals of $K(X, Y)$ and $X \tilde{\otimes}_{\varepsilon} Y$ ), and derive a kind of Rainwater result for the operator space $L_{e}\left(X_{c}^{\prime}, Y\right)$.

In Section 3 we discuss weak sequential completeness and reflexivity of $L_{e}\left(X_{c}^{\prime}, Y\right)$ and specialize the results to various spaces of compact operators.

In Section 4 the results considered so far are applied to spaces of vector-valued functions.

In [16], the results of this paper are being supplemented by determining concrete representations for the dual space of $L_{e}\left(X_{c}^{\prime}, Y\right)$ in terms of the duals $X^{\prime}$ and $Y^{\prime}$ of the factor spaces $X$ and $Y$.

0.2 Notation, terminology, and preliminary results. As far as locally convex duality is concerned, the terminology is that of J. Horvath's book [33], with the following exceptions: given a locally convex space $X$ (always assumed to be Hausdorff), we denote by $X_{c}^{\prime}, X_{\tau}^{\prime}$, and $X_{b}^{\prime}$ the topological dual $X^{\prime}$ of $X$ with the topology of uniform convergence on the compact, the weakly compact, and the bounded disks in $X$, respectively. (A disk is a convex circled set.) Accordingly, $X_{c}^{\prime \prime}, X_{\tau}^{\prime \prime}$, and $X_{b}^{\prime \prime}$ will denote the second adjoint $X^{\prime \prime}=\left(X_{b}^{\prime}\right)^{\prime}$ of $X$ with the topology of uniform convergence on the corresponding subsets of $X_{b}^{\prime}$. The bilinear form associated with the dual pair $\left(X, X^{\prime}\right)$ (evaluation of $x^{\prime} \in X^{\prime}$ at $\left.x \in X\right)$ will be denoted by $\left(x, x^{\prime}\right)$.

Given a subset $A$ of a locally convex space, its closed convex (resp. convex circled) hull will be denoted by $\bar{c} A$ (resp. $\overline{a c} A$ ), and the set of its extreme points by ext $A$.

Given two locally convex spaces $X$ and $Y$, a continuous linear operator $h: X \rightarrow Y$ is called [weakly] compact if there exists a zero neighbourhood $U$ in $X$ such that $h(U)$ is [weakly] relatively compact in $Y$.

Spaces of linear operators: $L(X, Y), W(X, Y)$, and $K(X, Y)$ denote the spaces of all continuous, all weakly compact, and all compact linear operators from $X$ into $Y . W^{b}(X, Y)$ (resp. $\left.K^{b}(X, Y)\right)$ is the space of all weakly continuous linear operators from $X$ into $Y$ which transform bounded sets into relatively weakly compact (resp. compact) subsets of $Y$. Usually, these operator spaces will be endowed with the topology of uniform convergence on the bounded subsets of $X$ (the operator norm, whenever $X$ and $Y$ are Banach spaces), and then denoted by $L_{b}(X, Y)$, $W_{b}(X, Y), K_{b}(X, Y)$ etc. $L_{c}(X, Y)$ will denote the space $L(X, Y)$ with the topology of uniform convergence on the compact disks in $X$.

Special spaces: A locally convex space will be called semi-reflexive (resp. semi-Montel) whenever all its bounded subsets are relatively weakly 
compact (resp. compact). Of particular importance will be a class of generalized $D F$ spaces: $X$ is said to be a $g D F$ space (generalized $D F$ ) [47, 48] if (i) its strong dual $X_{b}^{\prime}$ is a Fréchet space, and (ii) its topology is localizable on the bounded sets, i.e. linear operators into other locally convex spaces are continuous as soon as their restrictions to the bounded sets are. This class of spaces has been introduced in [40] and investigated by the second named author in $[46,47,48]$ in connection with function spaces with R. C. Buck's strict topology $[12,13]$.

[Weakly] compact operators on gDF spaces: The following general results of $[\mathbf{4 6 , 4 8}$ ] on [weakly] compact operators will be needed throughout the paper: [48, Thm. 3.1]: Given a gDF space $X$ and a Fréchet space $Y$, a weakly continuous linear operator from $X$ into $Y(i)$ is compact as soon as it transforms bounded sets into relatively compact ones, and (ii) is weakly compact as soon as it transforms bounded sets into weakly relatively compact ones, and, in addition, it is continuous for the original topologies of $X$ and $Y$ (for instance, if $X$ is Mackey). More generally: $W^{b}(X, Y)=$ $W(X, Y)$, whenever all weakly $\left(\sigma\left(X^{\prime}, X^{\prime \prime}\right)\right)$ compact disks of $X_{b}^{\prime}$ are equicontinuous.

\subsection{The $\varepsilon$-product of L. Schwartz.}

0.1 Definition $([53,54])$. Given two locally convex spaces $X$ and $Y$, the $\varepsilon$-product $X \varepsilon Y$ of $X$ and $Y$ is defined to be the space $L_{e}\left(X_{c}^{\prime}, Y\right)$ of continuous linear operators from $X_{c}^{\prime}$ into $Y$, endowed with the topology of uniform convergence on the equicontinuous subsets of $X^{\prime}$.

Four of the basic examples of (subspaces of) $\varepsilon$-products to be discussed in this paper are presented in the following table.

0.2 EXAMPLE: Spaces of compact linear operators

\begin{tabular}{c|l} 
(a) $K_{b}(X, Y)=L_{e}\left(X_{c}^{\prime \prime}, Y\right)=\left(X_{b}^{\prime}\right) \varepsilon Y$ & $X$ normed, \\
$u \mapsto u^{\prime \prime}$ & $Y$ Banach \\
\hline (b) $K_{b}(X, Y) \cong L_{e}\left(X_{c}^{\prime \prime}, Y\right) \cong\left(X_{b}^{\prime}\right) \varepsilon Y$ & $X$ gDF, \\
$u \mapsto u^{\prime \prime}$ & $Y$ Fréchet \\
\hline (c) $K_{b}^{b}(X, Y) \cong L_{e}\left(X_{c}^{\prime \prime}, Y\right) \cong\left(X_{b}^{\prime}\right) \varepsilon Y$ & $X, Y$ locally convex \\
$u \mapsto u^{\prime \prime}$ & $Y$ quasi-complete \\
\hline
\end{tabular}

(d) Given Banach spaces $X$ and $Y$, the $\varepsilon$-product $X_{\varepsilon} Y=L_{e}\left(X_{c}^{\prime}, Y\right)$ is exactly the space of compact weak*-weakly continuous linear operators from $X^{\prime}$ into $Y$, with the usual operator norm. 
0.3 EXAMPLE: Spaces of continuous linear operators

\begin{tabular}{|c|c|}
\hline $\begin{array}{c}L_{c}(X, Y) \stackrel{\widetilde{c}}{\leftrightarrows} L_{e}\left(\left(X_{c}^{\prime}\right)_{c}^{\prime}, Y\right) \cong\left(X_{c}^{\prime}\right) \varepsilon Y \\
u \mapsto u^{\prime \prime}\end{array}$ & $\begin{array}{l}X \text { and } Y \text { locally } \\
\text { convex }\end{array}$ \\
\hline $\begin{array}{l}\text { 0.4 EXAMPLE: Injective tensor products } \\
\qquad \begin{array}{r}X \tilde{\otimes}_{\varepsilon} Y \stackrel{\sim}{\hookrightarrow} L_{e}\left(X_{c}^{\prime}, Y\right) \cong X \varepsilon Y \\
x \otimes y \mapsto\left\{x^{\prime} \mapsto\left(x, x^{\prime}\right) y\right\}\end{array}\end{array}$ & $\begin{array}{l}X \text { and } Y \text { complete } \\
\text { locally convex }\end{array}$ \\
\hline $\begin{array}{c}\text { 0.5 EXAMPLE: Linearization of vector-valued functions } \\
C(S, X)_{\mathrm{co}} \cong L_{e}\left(X_{c}^{\prime}, C(S)_{\mathrm{co}}\right) \cong\left(C(S)_{\mathrm{co}}\right) \varepsilon X \\
F \mapsto F^{*}:\left\{x^{\prime} \mapsto x^{\prime} \circ F\right\}\end{array}$ & $\begin{array}{l}X \text { quasi-complete } \\
\text { locally convex }\end{array}$ \\
\hline $\begin{array}{c}C_{0}(S, X)_{\infty} \cong L_{e}\left(X_{c}^{\prime}, C_{0}(S)_{\infty}\right) \cong\left(C_{0}(S)_{\infty}\right) \varepsilon X \\
F \mapsto F^{*}:\left\{x^{\prime} \mapsto x^{\prime} \circ F\right\}\end{array}$ & $\begin{array}{l}S \text { locally compact } \\
\text { Hausdorff }\end{array}$ \\
\hline
\end{tabular}

We quote now from L. Schwartz' paper [54] the basic general facts on $\varepsilon$-products which will be needed throughout the paper.

(0.6.0) If $h$ is a linear operator from $X^{\prime}$ into $Y$, then we have: $h$ belongs to $X \varepsilon Y$, i.e. is continuous from $X_{c}^{\prime}$ into $Y$, if (and only if) it is weak*-weakly $\left(\sigma\left(X^{\prime}, X\right)-\sigma\left(Y, Y^{\prime}\right)\right)$ continuous and transforms the equicontinuous subsets of $X^{\prime}$ into relatively compact subsets of $Y$; equivalently, if (and only if) it is weak ${ }^{*}$-weakly continuous and its restrictions to the equicontinuous subsets of $X^{\prime}$ are continuous from $X_{c}^{\prime}$ into $Y$.

(0.6.1) If $X$ and $Y$ are quasi-complete (resp. complete), then $X \varepsilon Y$ is quasi-complete ( resp. complete). ([54, p. 29])

(0.6.2) If $X_{0}$ and $Y_{0}$ are linear subspaces of $X$ and $Y$, respectively, then $X_{0} \varepsilon Y_{0}$ is a topological linear subspace of $X \varepsilon Y .([\mathbf{5 4}, p .22]$.)

(0.6.3) The correspondence between $h \in L\left(X_{c}^{\prime}, Y\right)$ and its adjoint $h^{\prime}$ sets up a topological linear isomorphism between $X \varepsilon Y$ and $Y \varepsilon X$ :

$$
\begin{gathered}
X \varepsilon Y=L_{e}\left(X_{c}^{\prime}, Y\right) \cong L_{e}\left(Y_{c}^{\prime}, X\right)=Y \varepsilon X \\
h \mapsto h^{\prime}
\end{gathered}
$$

1. Weak compactness and weak conditional compactness in spaces of operators. In this section we develop the fundamental results on weak convergence in the operator space $L_{e}\left(X_{c}^{\prime}, Y\right)$. We also discuss weak conditional compactness, i.e. the problem of when a given sequence of 
operators in $L_{e}\left(X_{c}^{\prime}, Y\right)$ has a weak Cauchy subsequence. The results are then specified to spaces of compact operators and injective tensor products. Applications to vector-valued functions are given in $\$ 4$.

1.A General results. The key tool for an understanding of weak convergence in $L_{e}\left(X_{c}^{\prime}, Y\right)$ is provided by Proposition 1.2. We use the following notation:

1.1 Notation. Let $\left(h_{\lambda}\right)_{\lambda}$ be a net of linear operators from $X^{\prime}$ into $Y$.

(a) $\left(h_{\lambda}\right)_{\lambda}$ is said to be wot-(weak operator topology) convergent to a linear operator $h: X^{\prime} \rightarrow Y$, if $\left(h_{\lambda} x^{\prime}\right)_{\lambda} \subset Y$ is weakly $\left(\sigma\left(Y, Y^{\prime}\right)\right)$ convergent to $h x^{\prime}$ for all $x^{\prime} \in X^{\prime}$.

(b) More generally, given subsets $M$ and $N$ of $X^{\prime}$ and $Y^{\prime},\left(h_{\lambda}\right)_{\lambda}$ is said to be $M \otimes N$-wot convergent to a linear operator $h: X^{\prime} \rightarrow Y$, if $\left(h_{\lambda} m, n\right)_{\lambda}$ converges to $(h m, n)$ for all $m \in M$ and all $n \in N$.

1.2 Proposition. Let $X$ and $Y$ be locally convex spaces, and $H$ a subset of $L\left(X_{c}^{\prime}, Y\right)$.

(a) $H$ is weakly relatively compact in $L_{e}\left(X_{c}^{\prime}, Y\right)$ if and only if it is weak operator topology relatively compact in $L\left(X_{c}^{\prime}, Y\right)$.

(b) $H$ is weakly relatively compact in $L_{e}\left(X_{c}^{\prime}, Y\right)$ if and only if

(i) $H\left(x^{\prime}\right)$ is weakly relatively compact in $Y$ for all $x^{\prime} \in X^{\prime}$, and

(ii) every operator $h: X^{\prime} \rightarrow Y$ which is the wot-limit of a net $\left(h_{\lambda}\right)_{\lambda} \subset H$ is contained in $L_{e}\left(X_{c}^{\prime}, Y\right)$.

As an immediate consequence, we arrive at a first definite result on weak sequential convergence.

1.3 THEOREM. Let $X$ and $Y$ be arbitrary locally convex spaces.

(a) A sequence $\left(h_{n}\right)_{n} \subset L\left(X_{c}^{\prime}, Y\right)$ converges weakly in $L_{e}\left(X_{c}^{\prime}, Y\right)$ to $h \in L\left(X_{c}^{\prime}, Y\right)$ if and only if $\left(h_{n} x^{\prime}\right)_{n} \subset Y$ converges weakly $\left(\sigma\left(Y, Y^{\prime}\right)\right)$ to $h x^{\prime}$ for all $X^{\prime} \in X^{\prime}$.

(b) A sequence $\left(h_{n}\right)_{n} \subset L\left(X_{c}^{\prime}, Y\right)$ is weakly Cauchy in $L_{e}\left(X_{c}^{\prime}, Y\right)$ if and only if $\left(h_{n} x^{\prime}\right)_{n} \subset Y$ is weakly $\left(\sigma\left(Y, Y^{\prime}\right)\right)$ Cauchy in $Y$ for all $x^{\prime} \in X^{\prime}$.

Note that proposition (b) of this result follows from (a) and the fact that a sequence $\left(z_{n}\right)_{n}$ in a locally convex space $Z$ is Cauchy if and only if $\left(z_{n_{k+1}}-z_{n_{k}}\right)_{k}$ is convergent to zero for all strictly increasing sequences $\left(n_{k}\right)_{k}$ of integers.

Proof of Proposition 1.2. Part (a): We show that wot-compactness implies weak compactness. Then the proofs of all other assertions are straightforward. The basic idea is to embed $L_{e}\left(X_{c}^{\prime}, Y\right)$ topologically into a 
product of $C(K)$-spaces. Given any $h \in L\left(X_{c}^{\prime}, Y\right)$, consider the canonical bilinear form $B_{h}$ on $X^{\prime} \times Y^{\prime}$ associated with $h$, defined by $B_{h}\left(x^{\prime}, y^{\prime}\right)=$ $\left(h x^{\prime}, y^{\prime}\right) . B_{h}$ has continuous restrictions to $\left(U^{0}\right.$, weak $\left.{ }^{*}\right) \times\left(V^{0}\right.$, weak $\left.{ }^{*}\right)$, and we have the following topological linear embedding:

$$
\begin{aligned}
& L_{e}\left(X_{c}^{\prime}, Y\right) \stackrel{\widetilde{a}}{a}\left\{C\left(U^{0} \times V^{0}\right) \mid U, V\right\} \\
& h \mapsto\left(B_{h} \mid U^{0} \times V^{0}\right)_{U, V},
\end{aligned}
$$

where $U$ and $V$ run through zero neighbourhood bases of $X$ and $Y$. Hence, according to Tychonov's Theorem, a subset $H$ of $L_{e}\left(X_{c}^{\prime}, Y\right)$ is weakly compact if and only if all sets $B_{H} \mid U^{0} \times V^{0}=\left\{B_{h}\left|U^{0} \times V^{0}\right| h \in H\right\}$ are weakly compact in $C\left(U^{0} \times V^{0}\right)$. But if $H$ is a wot-compact subset of $L\left(X_{c}^{\prime}, Y\right)$, then the set $B_{H} \mid U^{0} \times V^{0}$ is bounded and pointwise compact, hence weakly compact in $C\left(U^{0} \times V^{0}\right)$. Pointwise compactness being clear from the assumption of wot-compactness of $H$, the boundedness of $B_{H} \mid U^{0} \times V^{0}$ is a consequence of the Banach-Steinhaus Theorem: given any $x^{\prime} \in X^{\prime}$, the set $\left(H x^{\prime}, y^{\prime}\right)$ is a bounded set of scalars for all $y^{\prime} \in Y^{\prime}$. This shows that $H \mid X_{U^{0}}^{\prime}$ is a pointwise bounded, and thus norm bounded set of continuous linear operators from the Banach space $\left(X_{U^{0}}^{\prime}, U^{0}\right)$ (span of $U^{0}$ in $X^{\prime}$ with $U^{0}$ as norm unit ball) into $Y$. This implies the boundedness of $B_{H} \mid U^{0} \times V^{0}$, and the proof of part (a) is complete.

Part (b): Clearly, only sufficiency of the two conditions needs to be checked. Hence, assume that conditions (i) and (ii) are fulfilled. According to part (a), it is enough to show that $H$ is wot-relatively compact. We show that every net in $H$ has a wot-convergent subnet. By Tychonov's Theorem, the set $\pi\left\{\overline{H\left(x^{\prime}\right)} \mid x^{\prime} \in X^{\prime}\right\}$ is a weakly compact subset of $Y^{X^{\prime}}$ (here, $\overline{H\left(x^{\prime}\right)}$ denotes the weak closure of $H\left(x^{\prime}\right)$ in $\left.Y\right)$. Hence, if $\left(h_{\lambda}\right)_{\lambda} \subset H$ is a net in $H$, then the net $\left(\left(h_{\lambda} x^{\prime}\right)_{x^{\prime} \in X^{\prime}}\right)_{\lambda}$ has a $\sigma\left(Y^{X^{\prime}},\left(Y^{X^{\prime}}\right)^{\prime}\right)$-convergent subnet $\left(\left(h_{\gamma} x^{\prime}\right)_{x^{\prime} \in X^{\prime}}\right)_{\gamma}: \quad\left(\left(h_{\gamma} x^{\prime}\right)_{x^{\prime} \in X^{\prime}}\right)_{\gamma} \rightarrow\left(h x^{\prime}\right)_{x^{\prime} \in X^{\prime}} \in Y^{X^{\prime}}$ with respect to $\left(Y, \sigma\left(Y, Y^{\prime}\right)\right)^{X^{\prime}}$. This implies that $\left(h_{\gamma} x^{\prime}\right)_{\gamma} \subset Y$ converges weakly to $h x^{\prime}$ for all $x^{\prime} \in X^{\prime}$. According to (ii), $h$ is an element of $L\left(X_{c}^{\prime}, Y\right)$, and we just showed that $\left(h_{\gamma}\right)_{\gamma}$ is wot-convergent to $h$. This completes the proof.

Proposition 1.2 can be used to derive the results of [62] on the problem of whether weak compactness properties of factors $A \subset X$ and $B \subset Y$ are inherited by their formal tensor product $A \otimes B=\{a \otimes b \mid a \in$ $A, b \in B\}$, viewed as a subset of the injective tensor product $X \otimes_{\varepsilon} Y$. The heredity problem for weak compactness itself can even be solved in the most satisfactory way, eliminating the assumption of [62] that one of the spaces $X$ or $Y$ be Smulian (weak relative countable compactness implies weak relative sequential compactness): 
1.4 Theorem. Let $X$ and $Y$ be locally convex spaces. If $A$ and $B$ are weakly relatively compact in $X$ and $Y$, respectively, then $A \otimes B$ is weakly relatively compact in $X \otimes_{\varepsilon} Y$, and we have: $\overline{A \otimes B}=\bar{A} \otimes \bar{B}$ (for the respective weak closures).

$$
\begin{aligned}
& \text { Proof. The "tensor map" } \\
& \qquad \begin{array}{c}
T:(X, \text { weak }) \times(Y, \text { weak }) \rightarrow\left(L\left(X_{c}^{\prime}, Y\right), \text { wot }\right) \\
(x, y) \mapsto x \otimes y:\left\{x^{\prime} \mapsto\left(x^{\prime}, x\right) y\right\}
\end{array}
\end{aligned}
$$

is bilinear and continuous. Hence, given weakly relatively compact subsets $A$ of $X$ and $B$ of $Y$, the set $\bar{A} \otimes \bar{B}=T(\bar{A} \times \bar{B})$ is wot-compact, and thus, by Proposition 1.2(a), weakly compact in $X \otimes_{\varepsilon} Y$. The rest of the proof is now clear.

We now turn to the fundamental general result on weak conditional compactness.

1.5 Terminology. A subset $A$ of a locally convex space is said to be weakly conditionally compact, if every sequence in $A$ has a weak Cauchy subsequence.

Considering the operator space $L_{e}\left(X_{c}^{\prime}, Y\right)$, we first note that an obvious necessary condition for a subset $H$ of $L_{e}\left(X_{c}^{\prime}, Y\right)$ to be weakly conditionally compact is that both $H\left(x^{\prime}\right)$ and $H^{\prime}\left(y^{\prime}\right)$ be weakly conditionally compact in $Y$ and $X$, respectively, for all $x^{\prime} \in X^{\prime}$ and all $y \in Y^{\prime}$. We get sufficient conditions if we require that at least one of them be weakly relatively compact. Here is the precise result:

1.6 Proposition. Let $X$ and $Y$ be Fréchet spaces, and $H$ a subset of $L\left(X_{c}^{\prime}, Y\right)$. If

(i) $H\left(x^{\prime}\right)$ is weakly relatively compact in $Y$ for all $x^{\prime} \in X^{\prime}$, and

(ii) $H^{\prime}\left(y^{\prime}\right)$ is weakly conditionally compact in $X$ for all $y^{\prime} \in Y^{\prime}$, then $H$ is weakly conditionally compact in $L_{e}\left(X_{c}^{\prime}, Y\right)$.

Whenever $Y$ is a weakly sequentially complete Fréchet space, then conditions (i) and (ii) are also necessary for $H$ to be weakly conditionally compact in $L_{e}\left(X_{c}^{\prime}, Y\right)$.

Proof. Our proof is an adaptation of Lewis' proof for the corresponding result on $X \tilde{\otimes}_{\varepsilon} Y$ for $X$ and $Y$ Banach spaces [37, Thm. 1.3]. Assume that conditions (i) and (ii) on $H \subset L\left(X_{c}^{\prime}, Y\right)$ are fulfilled, and let $\left(h_{n}\right)_{n} \subset H$ be an arbitrary sequence in $H$. First, since $X$ and $Y$ are Fréchet spaces, it is easy to see that there exists a closed separable linear subspace $Y_{0}$ of $Y$ such that the ranges of all $h_{n}$ 's are contained in $Y_{0}$. The dual $Y_{0}^{\prime}$ of $Y_{0}$ is 
weak*- $\left(\sigma\left(Y_{0}^{\prime}, Y_{0}\right)\right.$-) separable. Let $\left(y_{n}^{\prime}\right)_{n}$ be a $\sigma\left(Y_{0}^{\prime}, Y_{0}\right)$-dense countable set in $Y_{0}^{\prime}$. Applying condition (ii) and a standard diagonalization argument, we arrive at a subsequence $\left(h_{n_{k}}^{\prime}\right)_{k}$ of $\left(h_{n}^{\prime}\right)_{n}$ with the property that $\left(h_{n_{k}}^{\prime}\left(y_{j}^{\prime}\right)\right)_{k}$ is weakly Cauchy in $X$ for all $j$. Let now $x^{\prime} \in X^{\prime}, y_{0}^{\prime} \in Y_{0}^{\prime}$, and $\varepsilon>0$. Then, according to (i) and the $\sigma\left(Y_{0}^{\prime}, Y_{0}\right)$-denseness of $\left(y_{n}^{\prime}\right)_{n}$ in $Y_{0}^{\prime}$, and thus Mackey-denseness of $\operatorname{span}\left(y_{n}^{\prime}\right)_{n}$ in $Y_{0}^{\prime}$, there exists $y^{\prime} \in \operatorname{span}\left(y_{n}^{\prime}\right)_{n}$ such that $\left|\left(h_{n_{k}} x^{\prime}, y^{\prime}-y_{0}^{\prime}\right)\right|<\varepsilon$ for all $k$. The fact that $y^{\prime}$ is of the form $y^{\prime}=\sum_{1}^{m} \alpha_{i} y_{i}^{\prime}$ implies that, for $k$ and 1 large enough,

$$
\begin{aligned}
& \left|\left(h_{n_{k}} x^{\prime}, y_{0}^{\prime}\right)-\left(h_{n_{l}} x^{\prime}, y_{0}^{\prime}\right)\right| \\
& \quad \leq\left|\left(h_{n_{k}} x^{\prime}, y_{0}^{\prime}-y^{\prime}\right)\right|+\left|\left(x^{\prime}, h_{n_{k}}^{\prime} y^{\prime}-h_{n_{l}}^{\prime} y^{\prime}\right)\right|+\left|\left(h_{n_{l}} x^{\prime}, y^{\prime}-y_{0}^{\prime}\right)\right| \leq 3 \varepsilon .
\end{aligned}
$$

This proves that $\left(h_{n_{k}} x^{\prime}, y_{0}^{\prime}\right)_{k}$ is Cauchy for all $x^{\prime} \in X^{\prime}$ and all $y_{0}^{\prime} \in Y_{0}^{\prime}$. Hence, according to Theorem $1.3,\left(h_{n_{k}}\right)_{k}$ is weakly Cauchy in $L_{e}\left(X_{c}^{\prime}, Y_{0}\right)$, and thus, by result (0.6.2), in $L_{e}\left(X_{c}^{\prime}, Y\right)$. This completes the proof.

We give now conditions on $X$ and $Y$ assuring that every bounded sequence in $L_{e}\left(X_{c}^{\prime}, Y\right)$ has a weak Cauchy subsequence.

\subsection{TheOREM. Let $X$ and $Y$ be Fréchet spaces such that}

(i) every bounded sequence in $X$ has a weak Cauchy subsequence, and

(ii) every separable linear subspace of $Y$ has a (strongly) separable dual. Then every bounded sequence in $L_{e}\left(X_{c}^{\prime}, Y\right)$ has a weak Cauchy subsequence.

In particular, under these assumptions, the spaces $L_{e}\left(X_{c}^{\prime}, Y\right)$ and $X \tilde{\otimes}_{\varepsilon} Y$ are reflexive if and only if they are weakly sequentially complete.

Note. The assumptions of Theorem 1.7 are fulfilled, whenever $X$ is a Banach space not containing (an isomorph of) $l^{1}$ [44], or a reflexive Fréchet space, and $Y$ is a Banach space whose dual has the Radon-Nikodym property [55], or $Y$ is a reflexive Fréchet space.

Proof of Theorem 1.7. Again, as in the proof of the foregoing result, given a bounded sequence $\left(h_{n}\right)_{n} \subset L_{e}\left(X_{c}^{\prime}, Y\right)$, there exists a closed separable linear subspace $Y_{0}$ of $Y$ such that the ranges of all $h_{n}$ 's are contained in $Y_{0}$. According to assumption (ii), $Y_{0}^{\prime}$ is strongly separable. Let $\left(y_{n}^{\prime}\right)_{n}$ be a strongly dense subset. Applying assumption (i) and, again, a diagonalization argument, we arrive at a subsequence $\left(h_{n_{k}}\right)_{k}$ of $\left(h_{n}\right)_{n}$ such that $\left(h_{n_{k}}^{\prime}\left(y_{j}^{\prime}\right)\right)_{k}$ is weakly Cauchy in $X$ for all $j$. Taking adjoints again, this implies that, given $x^{\prime} \in X^{\prime}$, the sequence $\left(h_{n_{k}} x^{\prime}\right)_{k}$ is $\sigma\left(Y_{0},\left(y_{n}^{\prime}\right)_{n}\right)$-Cauchy. But, on bounded subsets of $Y_{0}, \sigma\left(Y_{0},\left(y_{n}^{\prime}\right)_{n}\right)$ and $\sigma\left(Y_{0}, Y_{0}^{\prime}\right)$ coincide. Hence, $\left(h_{n_{k}} x^{\prime}\right)_{k}$ is $\sigma\left(Y_{0}, Y_{0}^{\prime}\right)$-Cauchy in $Y_{0}$, and thus, according to Theorem 1.3, $\left(h_{n_{k}}\right)_{k}$ is weakly Cauchy in $L_{e}\left(X_{c}^{\prime}, Y_{0}\right)$. An appeal to result (0.6.2) completes the proof. 
We now discuss various special cases of the results given so far.

1.B Spaces of compact operators. Throughout this paragraph, we use the representation $K_{b}^{b}(X, Y) \cong L_{e}\left(X_{c}^{\prime \prime}, Y\right)$ of Example $0.2(\mathrm{c})$.

1.8 TheOREM. Let $X$ be a quasi-complete locally convex space, $S$ a locally compact Hausdorff space, and denote, as usual, by $C_{0}(S)$ the space of continuous scalar-valued functions on $S$ vanishing at infinity, endowed with the sup-norm. For a sequence $\left(k_{n}\right)_{n} \subset K\left(C_{0}(S), X\right)$, the following are equivalent:

(a) $\left(k_{n}\right)_{n}$ converges weakly in $K_{b}\left(C_{0}(S), X\right)$ to zero.

(b) $\left(x^{\prime} \circ k_{n}\right)\left(f_{i}\right) \rightarrow 0$ uniformly in $n$ for all $x^{\prime} \in X^{\prime}$ and all $\left(f_{i}\right)_{i} \subset C_{0}(S)$ which are uniformly bounded and converge pointwise to zero, and $k_{n}(f) \rightarrow 0$ weakly in $X$ for all $f \in C_{0}(S)$.

(c) $\left(k_{n}^{\prime \prime}\left(\chi_{0}\right)\right)_{n}$ converges weakly in $X$ to zero for all open subsets $O$ of $S$.

This extends corresponding results of Brooks/Lewis [11, Thms. 3.2 and 3.3]. It is a consequence of Theorem 1.3, the topological linear isomorphism $K_{b}\left(C_{0}(S), X\right) \cong L_{e}\left(M_{b}(S)_{c}^{\prime}, X\right)$ of $\S 0.3$, Example $0.2(\mathrm{c})$, and the criteria for weak convergence of sequences in $M_{b}(S)=\left(C_{0}(S)\right)_{b}^{\prime}$, cf. [29, V.4].

1.9 THEOREM. Let $X$ and $Y$ be locally convex spaces, $Y$ quasi-complete. For a subset $H$ of $K^{b}(X, Y)$, the following are equivalent:

(i) $H$ is weakly relatively compact in $K_{b}^{b}(X, Y)$.

(ii) The set $H^{\prime \prime}$ of second adjoints of members of $H$ is wot-relatively compact in $L\left(X_{c}^{\prime \prime}, Y\right)$.

(iii) (a) $H^{\prime \prime}\left(x^{\prime \prime}\right)$ is weakly relatively compact in $Y$ for all $x^{\prime \prime} \in X^{\prime \prime}$, and

(b) every wot-limit $u$ : $X^{\prime \prime} \rightarrow Y$ of a net $\left(h_{\lambda}^{\prime \prime}\right)_{\lambda} \subset H^{\prime \prime}$ is contained in $L\left(X_{c}^{\prime \prime}, Y\right)$.

Note. For $X$ normed and $Y$ Banach, or, more generally, $X$ gDF and $Y$ Fréchet, $K_{b}^{b}(X, Y)$ is exactly the space $K_{b}(X, Y)$ of compact linear operators from $X$ into $Y$ with the usual operator topology, see the last paragraph of $\S 0.2$.

REMARKs. (a) Theorem 1.9 asserts that for the special case of Banach spaces $X$ and $Y$ with $X$ reflexive, weak compactness in $K(X, Y)$ is equivalent to weak operator topology compactness in $K(X, Y)$. It is instructive to note that this relation breaks down as soon as we go over from the space of compact operators to the space of weakly compact operators: For a separable Hilbert space $H$, every bounded sequence in 
$L(H)$ has a weak operator topology convergent subsequence (cf. [1, 34 Satz 1, p. 85]). Yet, as is well known (see also Theorem 3.7), for $H$ infinite-dimensional, $L(H)$ is not reflexive $(K(H)$ is not even a dual space, by a result of Schatten [52]).

(b) Theorem 1.9 is a consequence of Proposition 1.2.

(c) For the special case of Banach spaces $X$ and $Y$, the equivalence of propositions (i) and (ii) of Theorem 1.9 has been proved by Kalton [35, Thm. 1].

1.10 TheOREM. Let $X$ be a normed space and $Y$ a Banach space, or, more generally, $X g D F$ and $Y$ Fréchet, and let $H$ be a subset of $K(X, Y)$. If

(a) $H^{\prime \prime}\left(x^{\prime \prime}\right)$ is weakly relatively compact in $Y$ for all $x^{\prime \prime} \in X^{\prime \prime}$, and

(b) $H^{\prime}\left(y^{\prime}\right)$ is weakly conditionally compact in $X_{b}^{\prime}$ for all $y^{\prime} \in Y^{\prime}$, then $H$ is weakly conditionally compact in $K_{b}(X, Y)$.

This is a consequence of Proposition 1.6.

1.11 THEOREM. Let $X$ be a gDF space and Y a Fréchet space such that

(a) every bounded sequence in $X_{b}^{\prime}$ has a weak Cauchy subsequence, and

(b) every separable linear subspace of $Y$ has a (strongly) separable dual. Then every bounded sequence in $K_{b}(X, Y)$ has a weak Cauchy subsequence. In particular, under these assumptions, $K_{b}(X, Y)$ is reflexive if (and only if) it is weakly sequentially complete.

As a special case, we obtain:

1.12 Corollary. Let $X$ and $Y$ be Banach spaces such that

(a) $X^{\prime}$ does not contain $l^{1}$ and $Y^{\prime}$ has $R N P$, or

(b) $X^{\prime \prime}$ has $R N P$ and $Y$ does not contain $l^{1}$.

Then $K(X, Y)$ does not contain $l^{1}$.

This result slightly extends Theorem 3 of [21].

1.C Injective tensor products. Throughout this paragraph, we use the embedding $X \tilde{\otimes}_{\varepsilon} Y \widetilde{\hookrightarrow} L_{e}\left(X_{c}^{\prime}, Y\right)$ of Example 0.4 .

1.13 TheOREM. Let $X$ and $Y$ be Fréchet spaces and $H$ a subset of $X \tilde{\otimes}_{\varepsilon} Y . I f$

(a) $H\left(x^{\prime}\right)$ is weakly relatively compact in $Y$ for all $x^{\prime} \in X^{\prime}$, and

(b) $H^{\prime}\left(y^{\prime}\right)$ is weakly conditionally compact in $X$ for all $y^{\prime} \in Y^{\prime}$, then $H$ is weakly conditionally compact in $X \tilde{\otimes}_{\varepsilon} Y$.

This is a consequence of Proposition 1.6. 
1.14 THEOREM. Let $X$ and $Y$ be Banach spaces such that

(a) $X$ does not contain $l^{1}$, and $Y^{\prime}$ has $R N P$, or

(b) $X^{\prime}$ has $R N P$, and $Y$ does not contain $l^{1}$.

Then $X \tilde{\otimes}_{\varepsilon} Y$ and even the whole space $L\left(X_{c}^{\prime}, Y\right)$ of compact weak ${ }^{*}$-weakly continuous linear operators from $X^{\prime}$ into $Y$, with the operator norm, does not contain $l^{1}$.

This is a consequence of Theorem 1.7 and the isometry $L_{b}\left(X_{c}^{\prime}, Y\right)=$ $L_{b}\left(Y_{c}^{\prime}, X\right)$ of $(0.6 .3)$.

Note. In connection with Theorem 1.14 , it is interesting to note that $X=Y=J T$ the James Tree space gives an example of Banach spaces $X$ and $Y$ both not containing $l^{1}$ but whose completed injective tensor product $X \tilde{\otimes}_{\varepsilon} Y$ does contain $l^{1}$, cf. [49].

2. Weak sequential convergence and extreme points in duals of operator spaces. We first determine the form of the extreme points in the dual of $L_{e}\left(X_{c}^{\prime}, Y\right)$. This will enable us to deduce criteria for weak compactness and for weak sequential convergence in a unified manner without recourse to Theorem 1.3.

2.1 Proposition. Let $X$ and $Y$ be locally convex spaces with zero neighbourhood bases $\mathcal{Q}_{X}$ and $\mathcal{Q}_{Y}$.

(a) A fundamental system of equicontinuous subsets of $\left(L_{e}\left(X_{c}^{\prime}, Y\right)\right)^{\prime}$ is given by the family of bipolar sets $\left(U^{0} \otimes V^{0}\right)^{00}$ of the products $U^{0} \otimes V^{0}$, $U \in \mathcal{Q}_{X}$ and $V \in \mathcal{Q}_{Y}$. $q_{Y}$.

(b) $\operatorname{ext}\left(\left(U^{0} \otimes V^{0}\right)^{00}\right) \subset\left(\operatorname{ext} U^{0}\right) \otimes\left(\operatorname{ext} V^{0}\right)$ for all $U \in \mathcal{Q}_{X}$ and $V \in$

Proof. $X^{\prime} \otimes Y^{\prime}$ is a linear subspace of $\left(L_{e}\left(X_{c}^{\prime}, Y\right)\right)^{\prime}$ via the linear embedding $j: X^{\prime} \otimes Y^{\prime} \rightarrow\left(L_{e}\left(X_{c}^{\prime}, Y\right)\right)^{\prime}$

$$
\sum_{1}^{n} x_{i}^{\prime} \otimes y_{i}^{\prime} \mapsto\left\{h \mapsto \sum_{1}^{n}\left(h x_{i}^{\prime}, y_{i}^{\prime}\right)\right\} \text {. }
$$

Let $W\left(U^{0}, V\right)=\left\{h \in L\left(X_{c}^{\prime}, Y\right) \mid h\left(U^{0}\right) \subset V\right\}, U \in \mathcal{Q}_{X}, V \in \mathcal{Q}_{Y} U$ and $V$ closed convex circled, be a basic zero neighbourhood in $L_{e}\left(X_{c}^{\prime}, Y\right)$. Then we have:

$$
\begin{aligned}
\left(U^{0} \otimes V^{0}\right)^{0} & =\left\{h \in L\left(X_{c}^{\prime}, Y\right)|| h\left(U^{0} \otimes V^{0}\right) \mid \leq 1\right\} \\
& =\left\{h \in L\left(X_{c}^{\prime}, Y\right)||\left(h\left(U^{0}\right), V^{0}\right) \mid \leq 1\right\} \\
& =\left\{h \in L\left(X_{c}^{\prime}, Y\right) \mid h\left(U^{0}\right) \subset V\right\}=W\left(U^{0}, V\right) .
\end{aligned}
$$

This proves assertion (a). 
In order to prove (b), we first show that the set $U^{0} \otimes V^{0}$ is $\sigma\left(\left(L_{e}\right)^{\prime}, L_{e}\right)$-compact and then apply the Krein-Milman Theorem. Consider the map

$$
\begin{gathered}
j_{U, V}:\left(U^{0}, \text { weak }^{*}\right) \times\left(V^{0}, \text { weak }^{*}\right) \rightarrow\left(\left(L_{e}\left(X_{c}^{\prime}, Y\right)\right)^{\prime}, \text { weak }^{*}\right) \\
\left(x^{\prime}, y^{\prime}\right) \mapsto\left\{h \mapsto\left(h x^{\prime}, y^{\prime}\right)\right\} .
\end{gathered}
$$

We show that this map is continuous. Let $\left(x_{\lambda}^{\prime}, y_{\lambda}^{\prime}\right)_{\lambda} \subset U^{0} \times V^{0}$ converge weak $^{*} \times$ weak $^{*}$ to $\left(x^{\prime}, y^{\prime}\right) \in U^{0} \times V^{0}$. Given $h \in L\left(X_{c}^{\prime}, Y\right)$, we have:

$$
\begin{aligned}
& \left|j_{U, V}\left(x_{\lambda}^{\prime}, y_{\lambda}^{\prime}\right)(h)-j_{U, V}\left(x^{\prime}, y^{\prime}\right)(h)\right| \\
& \quad \leq\left|\left(h\left(x_{\lambda}^{\prime}-x^{\prime}\right), y_{\lambda}^{\prime}\right)\right|+\left|\left(h x^{\prime}, y_{\lambda}^{\prime}-y^{\prime}\right)\right| .
\end{aligned}
$$

Since, on equicontinuous sets, the $c$-topology and the weak*-topology coincide, the net $\left(h\left(x_{\lambda}^{\prime}-x^{\prime}\right)\right)_{\lambda}$ tends to zero in $Y$. Since all of $\left(y_{\lambda}^{\prime}\right)_{\lambda}$ is contained in $V^{0}$, we thus have that the first term on the right is small for $\lambda$ large enough. The same is true for the second term, for the net $\left(y_{\lambda}^{\prime}-y^{\prime}\right)_{\lambda}$ tends weak* to zero. This proves the assertion. According to the Krein-Milman Theorem, we now know that $\operatorname{ext}\left(U^{0} \otimes V^{0}\right)^{00} \subset\left(U^{0} \otimes V^{0}\right)$. It is now an easy algebraic computation to show that $\operatorname{ext}\left(U^{0} \otimes V^{0}\right)^{00} \subset$ $\left(\right.$ ext $\left.U^{0}\right) \otimes\left(\operatorname{ext} V^{0}\right)$. This completes the proof.

2.2 THEOREM. Let $X$ and $Y$ be Banach spaces.

(a) ext $B\left(K^{\prime}(X, Y)\right) \subset\left(\operatorname{ext} B\left(X^{\prime \prime}\right)\right) \otimes\left(\operatorname{ext} B\left(Y^{\prime}\right)\right)$

(b) $L_{b}\left(X_{c}^{\prime}, Y\right)$ is the space of compact weak*-weakly continuous linear operators from $X^{\prime}$ into $Y$, with the operator norm. We have:

$$
\text { ext } B\left(L_{b}^{\prime}\left(X_{c}^{\prime}, Y\right)\right) \subset\left(\operatorname{ext} B\left(X^{\prime}\right)\right) \otimes\left(\operatorname{ext} B\left(Y^{\prime}\right)\right) \text {. }
$$

(c) ext $B\left(\left(X \tilde{\otimes}_{\varepsilon} Y\right)^{\prime}\right) \subset\left(\operatorname{ext} B\left(X^{\prime}\right)\right) \otimes\left(\operatorname{ext} B\left(Y^{\prime}\right)\right)$.

Proposition (a) of this theorem has recently been proved by Fakhouri [20, Cor. 2(b)]. In the context of more general locally convex spaces, we note:

2.3 Theorem. (a) Let $X$ be a gDF space, $Y$ a Fréchet space, and let $W(B, V)=\{k \in K(X, Y) \mid k(B) \subset V\}$ be a basic zero neighbourhood in $K(X, Y), B$ a bounded subset of $X, V$ a zero neighbourhood in $Y$. Then we have: $\operatorname{ext}(W(B, V))^{0} \subset\left(\operatorname{ext} B^{00}\right) \otimes\left(\operatorname{ext} V^{0}\right)$.

(b) Let $X$ and $Y$ be locally convex spaces, and let $W\left(U^{0}, V\right)=\{h \in$ $\left.X \tilde{\otimes}_{\varepsilon} Y \mid h\left(U^{0}\right) \subset V\right\}$ be a basic zero neighbourhood in $X \tilde{\otimes}_{\varepsilon} Y, U$ (resp. $V$ ) a zero neighbourhood in $X$ (resp. $Y)$. Then we have: $\operatorname{ext}\left(W\left(U^{0}, V\right)\right)^{0} \subset$ $\left(\operatorname{ext} U^{0}\right) \otimes\left(\operatorname{ext} V^{0}\right)$.

Proposition (b) of this result has recently been proved by Floret [22, $8.12]$. 
Note. In [49] it will be shown that the containment relations in Proposition 2.1 and Theorems 2.2 and 2.3 actually can be replaced by equality.

We now turn to applications of Proposition 2.1.

2.4 TheOREM. Let $X$ and $Y$ be locally convex spaces, $h \in L\left(X_{c}^{\prime}, Y\right)$, and $\left(h_{n}\right)_{n}$ a sequence in $L\left(X_{c}^{\prime}, Y\right)$. The following are equivalent:

(i) $\left(h_{n}\right)_{n}$ converges weakly in $L_{e}\left(X_{c}^{\prime}, Y\right)$ to $h$.

(ii) $\left(h_{n} x^{\prime}\right)_{n}$ converges weakly in $Y$ to $h x^{\prime}$ for all $x^{\prime} \in X^{\prime}$.

(iii) $\left(h_{n}\right)_{n}$ is bounded in $L_{e}\left(X_{c}^{\prime}, Y\right)$, and $\left(h_{n} x^{\prime}, y^{\prime}\right)_{n}$ converges to $\left(h x^{\prime}, y^{\prime}\right)$ for all $x^{\prime} \in \operatorname{ext} U^{0}, y^{\prime} \in \operatorname{ext} V^{0}, U \in \mathcal{Q}_{X}, V \in \mathcal{Q}_{Y}$.

Note. Since, for $X$ and $Y$ complete, $X \tilde{\otimes}_{\varepsilon} Y$ is a topological linear subspace of $L_{e}\left(X_{c}^{\prime}, Y\right)$, Theorem 2.4 in particular describes weakly convergent (and weak Cauchy) sequences in the completed injective tensor product of locally convex spaces.

For the case of Banach spaces $X$ and $Y$, the respective criteria for $X \tilde{\otimes}_{\varepsilon} Y$ have been proved by Lewis [37]. Related results in the $X \tilde{\otimes}_{\varepsilon} Y$-setting are also to be found in [61].

2.5 THEOREM. Let $X$ and $Y$ be locally convex spaces, $Y$ quasi-complete, and let $h,\left(h_{n}\right)_{n} \subset K^{b}(X, Y)$. Then the following are equivalent:

(i) $\left(h_{n}\right)_{n}$ converges weakly in $K_{b}^{b}(X, Y)$ to $h$.

(ii) $\left(h_{n}^{\prime \prime} x^{\prime \prime}\right)_{n}$ converges weakly in $Y$ to $h^{\prime \prime} x^{\prime \prime}$ for all $x^{\prime \prime} \in X^{\prime \prime}$.

(iii) $\left(h_{n}\right)_{n}$ is bounded in $K_{b}^{b}(X, Y)$, and $\left(h_{n}^{\prime \prime} x^{\prime \prime}, y^{\prime}\right)_{n}$ converges to $\left(h^{\prime \prime} x^{\prime \prime}, y^{\prime}\right)$ for all $x^{\prime \prime} \in$ ext $B^{00}, y^{\prime} \in \operatorname{ext} V^{0}, B$ out of a fundamental family of bounded sets in $X, V$ out of a zero neighbourhood base in $Y$.

For Banach spaces $X$ and $Y$, Theorem 2.5 has recently been proved by Fakhouri [21]. Proposition 2.1 can be combined with results of Bourgain/Talagrand [10], De Wilde [17], Floret [22], and of Grothendieck [28] in order to derive further weak compactness criteria for special subsets of $L_{e}\left(X_{c}^{\prime}, Y\right)$. We single out several particular examples in Theorem 2.6. In the following theorems, ext $Z^{\prime}$ denotes the set

$$
\bigcup\left\{\operatorname{ext} W^{0} \mid W \in \mathcal{Q}_{Z}\right\} \text {. }
$$

2.6 TheOREM. Let $X$ and $Y$ be locally convex spaces with zero neighbourhood bases $\mathcal{Q}_{X}$ and $\mathcal{Q}_{Y}$, and let $H$ be a bounded subset of $L_{e}\left(X_{c}^{\prime}, Y\right)$.

(a) $H$ is weakly relatively sequentially compact in $L_{e}\left(X_{c}^{\prime}, Y\right)$ if and only if it is ext $X^{\prime} \otimes$ ext $Y^{\prime}$-wot relatively sequentially compact in $L\left(X_{c}^{\prime}, Y\right)$. 
(b) If, in addition, $X$ and $Y$ are quasi-complete, then $H$ is weakly relatively compact in $L_{e}\left(X_{c}^{\prime}, Y\right)$ if and only if it fulfills the ext $X^{\prime} \otimes$ ext $Y^{\prime}$ interchangeable double limits property: there exist no sequences $\left(h_{n}\right)_{n} \subset H$ and $\left(x_{m}^{\prime}\right)_{m} \subset \operatorname{ext} U^{0}$ and $\left(y_{m}^{\prime}\right)_{m} \subset \operatorname{ext} V^{0}, U \in \mathcal{Q}_{X}, V \in \mathcal{Q}_{Y}$, such that both limits $\lim _{n} \lim _{m}\left(h_{n} x_{m}^{\prime}, y_{m}^{\prime}\right)$ and $\lim _{m} \lim _{n}\left(h_{n} x_{m}^{\prime}, y_{m}^{\prime}\right)$ exist and are distinct.

(c) If, in addition, $X$ and $Y$ are complete, or if $H$ is convex and its closure is complete in the Mackey topology of $L_{e}\left(X_{c}^{\prime}, Y\right)$, then $H$ is weakly relatively compact in $L_{e}\left(X_{c}^{\prime}, Y\right)$ if and only if it is ext $X^{\prime} \otimes \operatorname{ext} Y^{\prime}$-wot relatively countably compact in $L\left(X_{c}^{\prime}, Y\right)$.

For proposition (b), consult [30, Thm. 7.b]. The first part of proposition (c) follows from a combination of Proposition 2.1 with an extension to locally convex spaces of Bourgain/Talagrand's result in [10] which states that a bounded subset of a Banach space $Z$ is weakly relatively compact if (and only if) it is ext $B_{Z}$-wot relatively countably compact. For the second part of proposition (c), consult [18, Prop. 5.1] and [22, 8.3].

Finally, a further special result can be derived from Proposition 2.1 and [27] if we consider the case that one of the factor spaces, $X$ say, has a zero neighbourhood base $\mathcal{Q}_{X}$ such that ext $U^{0} \cup\{0\}$ is a weak*-compact subset of $U^{0}$ for all $U \in \mathcal{Q}_{X}$. For instance, think of $X=(C(T)$, co), the space of continuous scalar-valued functions on a completely regular Hausdorff space $T$, with the compact-open topology (for further examples, see Theorem 4.3 in $\S 4$ ).

2.7 Proposition. Let $X$ and $Y$ be quasi-complete locally convex spaces such that $X$ has a zero neighbourhood base $\mathcal{Q}_{X}$ with the property that ext $U^{0} \cup\{0\}$ is a weak*-compact subset of $U^{0}$ for all $U \in \mathcal{Q}_{X}$. Then a subset $H$ of $L\left(X_{c}^{\prime}, Y\right)$ is weakly relatively compact in $L_{e}\left(X_{c}^{\prime}, Y\right)$ if and only if

(i) $H$ is bounded in $L_{e}\left(X_{c}^{\prime}, Y\right)$.

(ii) $H\left(x^{\prime}\right)$ is weakly relatively compact in $Y$ for all $x^{\prime} \in \operatorname{ext} U^{0}$, $U \in \mathcal{Q}_{X}$.

(iii) Every $\left(\right.$ ext $\left.X^{\prime}\right) \otimes Y^{\prime}$-wot Cauchy net in $H$ has an (ext $\left.X^{\prime}\right) \otimes Y^{\prime}$-wot limit in $L\left(X_{c}^{\prime}, Y\right)$.

Proof. According to [27, Thm. 7.a], for a proof of the sufficiency, we have to show that all sets $\left\{B_{h} \mid\right.$ ext $\left.U^{0} \cup\{0\} \times V^{0} \mid h \in H\right\}$ are weakly compact in $C\left(\operatorname{ext} U^{0} \cup\{0\} \times V^{0}\right), U \in \mathcal{Q}_{X}, V \in \mathcal{Q}_{Y}$. This can be proved by a technique analogous to the one of the proof of Proposition 1.2. 
As before, the last result can be interpreted for spaces of compact operators and injective tensor products. This is left to the interested reader. Applications to spaces of vector-valued functions will be given in $\S 4$.

3. Weak sequential completeness and reflexivity. Concentrating for the moment on the case of Fréchet spaces $X$ and $Y$, we first note that a trivial necessary condition for $L_{e}\left(X_{c}^{\prime}, Y\right)$ to be reflexive is that both $X$ and $Y$ be reflexive, for $X$ and $Y$ can be realized as closed linear subspaces of $L_{e}\left(X_{c}^{\prime}, Y\right)$. But, under these circumstances, namely $X$ and $Y$ reflexive Fréchet, we know already from Theorem 1.7 that $L_{e}\left(X_{c}^{\prime}, Y\right)$ is reflexive if only it is weakly sequentially complete. For this reason, we first study weak sequential completeness of $L_{e}\left(X_{c}^{\prime}, Y\right)$.

3.1 Proposition. Let $X$ and $Y$ be locally convex spaces. $L_{e}\left(X_{c}^{\prime}, Y\right)$ is weakly sequentially complete if and only if

(i) $X$ and $Y$ are weakly sequentially complete, and

(ii) $L\left(X_{c}^{\prime}, Y\right)$ is wot-sequentially closed in $L\left(X_{\tau}^{\prime}, Y\right)$.

ReMARKS. Recall that $X_{\tau}^{\prime}$ denotes the dual of $X$ with the Mackey topology $\tau\left(X^{\prime}, X\right)$ of uniform convergence on the weakly compact disks in $X$. Hence, $L\left(X_{\tau}^{\prime}, Y\right)$ is exactly the space of all weak*-weakly continuous linear operators from $X^{\prime}$ into $Y$. Proposition (ii) thus means that any weak*-weakly continuous linear operator from $X^{\prime}$ into $Y$ which is the wot-limit of a sequence of $c$-continuous linear operators necessarily must transform equicontinuous subsets of $X^{\prime}$ into subsets of $Y$ which are relatively compact for the original topology of $Y$.

For a proof of Proposition 3.1, we need the following technical result.

3.2 LemMA. If $X$ and $Y$ are locally convex spaces such that $X$ is weakly sequentially complete, then every wot-limit $h: X^{\prime} \rightarrow Y$ of a sequence $\left(h_{n}\right)_{n} \subset$ $L\left(X_{\tau}^{\prime}, Y\right)$ is contained in $L\left(X_{\tau}^{\prime}, Y\right)$.

Proof. Assuming that (i) $\left(h_{n} x^{\prime}, y^{\prime}\right) \rightarrow\left(h x^{\prime}, y^{\prime}\right)$ for all $x^{\prime} \in X^{\prime}, y^{\prime} \in Y^{\prime}$, and a sequence $\left(h_{n}\right)_{n} \subset L\left(X_{\tau}^{\prime}, Y\right)$, we have to show that $h$ is weak*-weakly continuous. First, our assumption implies that $\left(h_{n}^{\prime} y^{\prime}\right)_{n}$ is weakly Cauchy, and thus weakly convergent in $X$ for all $y^{\prime} \in Y^{\prime}$. This defines a linear map $v: Y^{\prime} \rightarrow X$ with the property that (ii) $\left(h_{n}^{\prime} y^{\prime}, x^{\prime}\right) \rightarrow\left(v y^{\prime}, x^{\prime}\right)$ for all $x^{\prime} \in X^{\prime}$, $y^{\prime} \in Y^{\prime}$. (i) and (ii) together show that (iii) $\left(h x^{\prime}, y^{\prime}\right)=\left(v y^{\prime}, x^{\prime}\right)$ for all $x^{\prime} \in X^{\prime}, y^{\prime} \in Y^{\prime}$. Hence, given a weak*-convergent net $\left(x_{\lambda}^{\prime}\right)_{\lambda} \rightarrow x^{\prime}$, and $y^{\prime} \in Y^{\prime}$, we have: (iv) $\left(h x_{\lambda}^{\prime}, y^{\prime}\right)=\left(v y^{\prime}, x_{\lambda}^{\prime}\right) \rightarrow\left(v y^{\prime}, x^{\prime}\right)=\left(h x^{\prime}, y^{\prime}\right)$, which proves our assertion. 
Proof of Proposition 3.1. Necessity of the conditions is a consequence of Theorem 1.3. For a proof of the sufficiency part, let $\left(h_{n}\right)_{n}$ be a weak Cauchy sequence in $L_{e}\left(X_{c}^{\prime}, Y\right)$. Then $\left(h_{n} x^{\prime}\right)_{n}$ is weakly Cauchy in $Y$, and thus, according to (i), weakly convergent in $Y$, for all $x^{\prime} \in X^{\prime}$. This defines a linear operator $v: X^{\prime} \rightarrow Y$ with the property that $\left(h_{n} x^{\prime}, y^{\prime}\right) \rightarrow\left(v x^{\prime}, y^{\prime}\right)$ for all $x^{\prime} \in X^{\prime}, y^{\prime} \in Y^{\prime}$. According to Lemma 3.2, $v$ belongs to $L\left(X_{\tau}^{\prime}, Y\right)$. An appeal to (ii) now completes the proof.

3.3 Proposition. (a) Given two complete locally convex spaces $X$ and $Y$, the space $X \tilde{\otimes}_{\varepsilon} Y$ is weakly sequentially complete if and only if both $X$ and $Y$ are weakly sequentially complete, and $X \tilde{\otimes}_{\varepsilon} Y$ is wot-sequentially closed in $L\left(X_{\tau}^{\prime}, Y\right)$.

(b) Given Banach spaces $X$ and $Y$, or, more generally, a gDF space $X$ and a Fréchet space $Y$, the space $K_{b}(X, Y)$ is weakly sequentially complete if and only if $X_{b}^{\prime}$ and $Y$ are weakly sequentially complete and $L\left(X_{c}^{\prime \prime}, Y\right)$ is wot-sequentially closed in $L\left(X_{\tau}^{\prime \prime}, Y\right)$.

REMARKS. Proposition (a) follows from Lemma 3.2 in the same way as Proposition 3.1. Proposition (b) is a special case of Proposition 3.1.

Proposition (a) shows that in the corresponding result of $[60, \mathrm{Thm}$. 3.2], the assumption of the approximation property is superfluous.

Condition (ii) of Proposition 3.1 is trivially fulfilled whenever $L\left(X_{c}^{\prime}, Y\right)$ and $L\left(X_{\tau}^{\prime}, Y\right)$ coincide. Hence, the condition $L\left(X_{c}^{\prime}, Y\right)=L\left(X_{\tau}^{\prime}, Y\right)$ (together with $X$ and $Y$ being weakly sequentially complete) is a simple, but seemingly quite strong sufficient condition for weak sequential completeness of $L_{e}\left(X_{c}^{\prime}, Y\right)$. It comes as a little surprise that, conversely, under the presence of the metric approximation property this condition is even necessary. The prototype result in this direction is that of Lewis [37, Thm. $2.1]$ in the context of Banach spaces. We extend Lewis' technique of proof to the Fréchet space setting. First, we recall Grothendieck's notion of the metric approximation property for locally convex spaces.

Terminology. A locally convex space $Z$ has the metric approximation property m.a.p. [30, p. 182] if there exists an equicontinuous subset $H$ of finite rank operators in $L(Z, Z)$ such that the identity map on $Z$ is contained in the $c$-closure of $H$.

3.4 THEOREM. Let $X$ and $Y$ be locally convex spaces.

(a) If $X$ and $Y$ are weakly sequentially complete, and $L\left(X_{\tau}^{\prime}, Y\right)=$ $L\left(X_{c}^{\prime}, Y\right)$, then $L_{e}\left(X_{c}^{\prime}, Y\right)$ is weakly sequentially complete. If, in addition, $X$ and $Y$ are complete, the same assertion holds for $X \tilde{\otimes}_{\varepsilon} Y$.

(b) Conversely, if $X$ and $Y$ are Fréchet spaces such that $X$ or $Y$ has m.a.p., then we have: If $L_{e}\left(X_{c}^{\prime}, Y\right)\left(=X \tilde{\otimes}_{\varepsilon} Y\right)$ is weakly sequentially 
complete, then $X$ and $Y$ are weakly sequentially complete, and $L\left(X_{\tau}^{\prime}, Y\right)=$ $L\left(X_{c}^{\prime}, Y\right)$ : every weak*-weakly continuous linear operator from $X^{\prime}$ into $Y$ transforms equicontinuous subsets of $X^{\prime}$ into relatively compact subsets of $Y$.

Note. The identity $L\left(X_{\tau}^{\prime}, Y\right)=L\left(X_{c}^{\prime}, Y\right)$ holds trivially whenever $X$ or $Y$ is semi-Montel (bounded sets are relatively compact), or whenever in $X$ or $Y$ the families of compact and of weakly compact subsets coincide. In particular, for $X$ or $Y$ a Banach space with the Schur property (weak and norm sequential convergence coincide). Thus, Theorem 3.4 extends corresponding results in [60], and the result of [38].

Proof of Proposition (b) of Theorem 3.4. According to the symmetry of the assertion, it is no restriction to assume that $Y$ has m.a.p. For a given $u \in L\left(X_{\tau}^{\prime}, Y\right)$, we have to show that $u\left(U^{0}\right)$ is relatively compact in $Y$ for all $U \in \mathcal{Q}_{X}$, i.e. that, given any sequence $\left(x_{n}^{\prime}\right)_{n} \subset U^{0}$, the sequence $\left(u x_{n}^{\prime}\right)_{n}$ has a convergent subsequence. Following Lewis' idea of proof [37, Thm. 2.1], this is done by showing that there exists $v \in L\left(X_{c}^{\prime}, Y\right)$ such that $u x_{n}^{\prime}=v x_{n}^{\prime}$. We reduce the Fréchet space case to a Banach space situation by means of the following facts: first, since $X_{\tau}^{\prime}$ is a semi-reflexive gDF space, the operator $u$ actually is weakly compact [48, Thm. 2.3], i.e. it transforms a certain $\tau\left(X^{\prime}, X\right)$-zero neighbourhood into a weakly compact disk $C$ in $Y . Y$ being a Fréchet space, there exists a weakly compact disk $D$ in $Y$ such that the Banach space $\left(Y_{D}, D\right)$ (the span of $D$ in $Y$ with norm unit ball $D$ ) induces the same topology on $C$ as the original topology of $Y$ [48, Lemma in $\S 1]$. In particular, $C$ is a weakly compact disk in $\left(Y_{D}, D\right)$. Hence, the closure $W$ of $Y_{C}=\operatorname{span} C$ in $\left(Y_{D}, D\right)$ is a weakly compactly generated Banach space, and the closed linear span $Z_{0}$ of $\left(u x_{n}^{\prime}\right)_{n}$ in $(W, D)$ is a separable linear subspace. Returning now to Lewis' arguments, we conclude by a result of Amir/Lindenstrauss [2] that there exists a closed separable linear subspace $Z$ of $(W, D), Z_{0} \subset Z \subset W$, and a norm one projection $P: W \rightarrow Z$. Let $\left(y_{n}\right)_{n}$ be a dense countable subset of $Z$. We now use the m.a.p. of $Y$ to assert the existence of an equicontinuous sequence of finite rank operators $\left(h_{n}\right)_{n} \subset L(Y, Y)$ with the property that $\left(\mathrm{id}_{Y}-h_{n}\right)\left(a c\left\{y_{1}, \ldots, y_{n}\right\}\right) \subset V_{n} / n$, where $\left(V_{n}\right)_{n}$ is a decreasing zero neighbourhood base in $Y$, all $V_{n}$ closed convex circled. Denote by $v_{n}$ the composition

$$
v_{n}: X^{\prime} \stackrel{u}{\rightarrow}(W, D) \stackrel{P}{\rightarrow} Z \stackrel{h_{n} \mid Z}{\rightarrow} Y .
$$

The $v_{n}$ are continuous and of finite rank, hence $\left(v_{n}\right)_{n} \subset L\left(X_{c}^{\prime}, Y\right)$. We now show that $v_{n} x^{\prime} \rightarrow(P \circ u)\left(x^{\prime}\right)$ in $Y$ for all $x^{\prime} \in X^{\prime}$. Let $x^{\prime} \in X^{\prime}$, and $m$ arbitrary. 
$n$.

(i) There exists $V \in \mathcal{Q}_{Y}, V \subset \frac{1}{3} V_{m}$, such that $h_{n}(V) \subset V_{m} / 3$ for all

(ii) There exists $k$ such that $(P \circ u)\left(x^{\prime}\right)-y_{k} \in V$. Hence, if $n_{0}=$ $\max \{m, k, 3\}$, we have:

(iii) $\left(h_{n}-\mathrm{id}_{Y}\right)\left(a c\left\{y_{1}, \ldots, y_{n_{0}}\right\}\right) \subset V_{n} / n \subset V_{n_{0}} / n_{0} \subset V_{m} / 3$ for all $n \geq$ $n_{0}$. Altogether, this implies:

(iv) $v_{n} x^{\prime}-(P \circ u)\left(x^{\prime}\right)=\left(h_{n} \circ P \circ u\right)\left(x^{\prime}\right)-P \circ u\left(x^{\prime}\right)=$ $h_{n}\left((P \circ u)\left(x^{\prime}\right)-y_{k}\right)+\left(h_{n} y_{k}-y_{k}\right)+\left(y_{k}-P \circ u\left(x^{\prime}\right)\right) \in V_{m}$ for all $n \geq$ $n_{0}$. In particular, according to Theorem $1.3,\left(v_{n}\right)_{n}$ is weakly Cauchy in $L_{e}\left(X_{c}^{\prime}, Y\right)$, and thus, by assumption, tends weakly to $v \in L\left(X_{c}^{\prime}, Y\right)$. Together with the fact $v_{n} x^{\prime} \rightarrow P \circ u\left(x^{\prime}\right)$ in $Y$ for all $x^{\prime} \in X^{\prime}$, we conclude that $v x^{\prime}=(P \circ u)\left(x^{\prime}\right)$ for all $x^{\prime} \in X^{\prime}$, and, in particular, $v x_{n}^{\prime}=$ $(P \circ u)\left(x_{n}^{\prime}\right)=u x_{n}^{\prime}$ for all $n$. This completes the proof.

A variety of consequences of Theorem 3.4 follows.

3.5 THEOREM. Let $X$ and $Y$ be Banach spaces.

(a) If $X^{\prime}$ and $Y$ are weakly sequentially complete, and every weakly compact linear operator from $X$ into $Y$ is compact, then $K_{b}(X, Y)$ is weakly sequentially complete.

(b) Conversely, if $X^{\prime}$ or $Y$ has m.a.p., and $K_{b}(X, Y)$ is weakly sequentially complete, then $X^{\prime}$ and $Y$ are weakly sequentially complete, and every weakly compact linear operator from $X$ into $Y$ is compact.

3.6 CorollaRY. Whenever $X$ is a Banach space whose dual is weakly sequentially complete, and $Y$ is a Banach space with the Schur property, then $K_{b}(X, Y)$ is weakly sequentially complete.

Because of the many interesting concrete gDF spaces in analyis, like spaces of bounded continuous functions $C_{b}(S)_{\beta}, S$ locally compact Hausdorff, or of bounded holomorphic functions $H^{\infty}(G)_{\beta}, G$ a plane region, both with the strict topology $\beta$ of $\mathrm{R}$. C. Buck $[12,13]$ (cf. [15] for a survey), we also note a version of Theorem 3.5 for the gDF- $F$-situation.

3.7 THEOREM. Let $X$ be a gDF space, and $Y$ a Fréchet space such that $X_{b}^{\prime}$ and $Y$ are weakly sequentially complete. Then we have:

(a) If $W^{b}(X, Y)=K(X, Y)$, then $K_{b}(X, Y)$ is weakly sequentially complete.

(b) Conversely, if $X_{b}^{\prime}$ or $Y$ has m.a.p., and $K_{b}(X, Y)$ is weakly sequentially complete, then $W^{b}(X, Y)=K(X, Y)$.

Note. Whenever $X$ is a Mackey gDF space, then $W^{b}(X, Y)=$ $W(X, Y)$, and whenever $X$ is gDF and $Y$ Fréchet such that $X$ or $Y$ is semi-reflexive, then $L(X, Y)=W(X, Y)$ (see the last paragraph of $\S 0.2$ ). 
3.8 COROLlaRY. (a) Whenever $S$ is a locally compact Hausdorff space, and $X$ a Banach space with the Schur property, or a Fréchet-Montel space, then the space $K_{b}\left(C_{b}(S)_{\beta}, X\right)$ is weakly sequentially complete. In particular, this is true for $K_{b}(C(K), X), K$ compact Hausdorff, $X$ as before.

(b) Whenever $Y$ is a sequentially complete Fréchet space, then $L_{b}\left(H^{\infty}(D)_{\beta}, Y\right)=K_{b}\left(H^{\infty}(D)_{\beta}, Y\right)$ ( $D$ the open unit disk in the complex plane) is weakly sequentially complete.

It is a long standing conjecture that, for reflexive Banach spaces $X$ and $Y$, the space $L(X, Y)$ is reflexive if and only if all bounded linear operators from $X$ into $Y$ are compact. In a series of papers, Ruckle [45], Holub [32], and Kalton [35] established this result under the additional assumption that $X$ or $Y$ has a.p. We derive this (and a little bit more) from Theorem 3.7 and Theorem 1.11 in the more general gDF-F-setting. (For further information on the above conjecture, see also Cor. 3.3 of our paper [16].)

3.9 THEOREM. Let $X$ be a Mackey gDF space, and $Y$ a Fréchet space such that $X_{b}^{\prime}$ and $Y$ are reflexive. Consider the following statements:

(a) $L(X, Y)=K(X, Y)$,

(b) $L_{b}(X, Y)$ is reflexive,

(c) $K_{b}(X, Y)$ is reflexive,

(d) $K_{b}(X, Y)$ is weakly sequentially complete.

Then (a) implies (b), (b) implies (c), and (c) is equivalent to (d). Finally, whenever $X_{b}^{\prime}$ or $Y$ has m.a.p., then (d) implies (a).

We are now in a position to combine Theorems 1.7 and 3.4 to conclude that for two reflexive Fréchet spaces $X$ and $Y$, one of which is semi-Montel, the space $L_{e}\left(X_{c}^{\prime}, Y\right)$ is a reflexive Fréchet space. However, with a little additional effort, we are able to show that the semi-reflexivity part of this result is true for any locally convex spaces. We need the following technical result which is a kind of "net-version" of Lemma 3.2.

3.10 Lemma. Let $X$ and $Y$ be locally convex spaces such that $X$ is semi-reflexive, and let $H$ be a bounded subset of $L_{e}\left(X_{\tau}^{\prime}, Y\right)$. Then every wot-limit $h: X^{\prime} \rightarrow Y$ of a net $\left(h_{\lambda}\right)_{\lambda} \subset H$ is contained in $L\left(X_{\tau}^{\prime}, Y\right)$.

A combination of Lemma 3.10 and Proposition 1.2 directly leads to the fundamental result on the semi-reflexivity of $L_{e}\left(X_{c}^{\prime}, Y\right)$.

3.11 TheOREM. Let $X$ and $Y$ be semi-reflexive locally convex spaces such that $L\left(X_{\tau}^{\prime}, Y\right)=L\left(X_{c}^{\prime}, Y\right)$. Then the space $L_{e}\left(X_{c}^{\prime}, Y\right)$ and, in case $X$ and $Y$ are complete, also the space $X \tilde{\otimes}_{\varepsilon} Y$ is semi-reflexive. 
3.12 Corollary. Let $X$ and $Y$ be semi-reflexive locally convex spaces one of which is semi-Montel. Then the space $L_{e}\left(X_{c}^{\prime}, Y\right)$ and, in case $X$ and $Y$ are complete, also the space $X \tilde{\otimes}_{\varepsilon} Y$ is semi-reflexive.

REMARKS. (a) Corollary 3.12 improves a result of Grothendieck [30, Cor. p. 118] in that it shows that not the full Schwartz property is needed in one of the factors $X$ or $Y$, but only the semi-Montel property.

(b) Theorem 3.11 exposes the matter of semi-reflexivity of $X \tilde{\otimes}_{\varepsilon} Y$ in full generality. In particular, it extends corresponding results in [63] and eliminates superfluous restrictions.

We now derive further consequences of Theorem 3.11.

3.13 TheOREM. Let $X$ be a gDF space and $Y$ a Fréchet space such that $X_{b}^{\prime}$ and $Y$ are reflexive. If $L\left(X_{\tau}^{\prime \prime}, Y\right)=L\left(X_{c}^{\prime \prime}, Y\right)$, in particular, if $X_{b}^{\prime}$ or $Y$ is semi-Montel, then $K_{b}(X, Y)$ is a reflexive Fréchet space.

3.14 THEOREM. (a) Whenever $X$ is a barrelled locally convex space, then $L_{c}(X, Y)$ is semi-reflexive if and only if $Y$ is semi-reflexive.

(b) Whenever $X$ is a Montel space, then $L_{b}(X, Y)$ is semi-reflexive if and only if $Y$ is semi-reflexive.

Theorem 3.14 extends corresponding results in [63, Cor. 3.8] and eliminates superfluous restrictions.

Proof of Theorem 3.14. Whenever $X$ is barrelled, then $X_{c}^{\prime}$ is semiMontel. Thus, Proposition (a) follows from Corollary 3.12 and the representation $L_{c}(X, Y)=L_{e}\left(\left(X_{c}^{\prime}\right)_{c}^{\prime}, Y\right)$ of Example 0.3. Proposition (b) is a special case of (a).

4. Weak compactness in spaces of vector-valued functions. This section is devoted to a study of weak compactness in various spaces of vector-valued functions. Starting from the principle of linearizing vectorvalued functions, as indicated in Example 0.5, the criteria will turn out to be direct consequences of our general results in the context of the operator space $L_{e}\left(X_{c}^{\prime}, Y\right)$.

We place our results in the general context of weighted spaces of vector-valued continuous functions. These spaces have been introduced in the scalar case by L. Nachbin [39], and studied in the vector-valued case by K.-D. Bierstedt $[4,5]$ and J. B. Prolla [42]. We recall the basic definition, and refer the reader for details to Bierstedt's surveys [6, 7] and his paper [8]. 
Definition. Let $T$ be a completely regular Hausdorff space, $X$ a locally convex space, and $V \neq \varnothing$ a directed family of weights on $T$ (real-valued non-negative upper semicontinuous functions on $T$ ). Then the weighted space $C V_{0}(T, X)$ is defined as follows: $C V_{0}(T, X)=\{F$ : $T \rightarrow X$ continuous $\mid v F: t \mapsto v(t) F(t)$ vanishes at infinity for all $v \in V\}$, endowed with the locally convex topology generated by the semi-norms $\left\{b_{v, q}\right\}, v \in V, q$ a continuous semi-norm on $X$, where $b_{v, q}(F)=$ $\sup \{v(t) q(F(t)) \mid t \in T\}$. (A function $F: T \rightarrow X$ is said to vanish at infinity if, for every continuous seminorm $q$ on $X$, and every $\varepsilon>0$, there exists a compact subset $K$ of $T$ such that $q(F(t))<\varepsilon$ for all $t \in T \backslash K$.)

\subsection{EXAMPles. (cf. $[4,5,6])$}

\begin{tabular}{l|l}
\multicolumn{1}{c|}{ Weights } & \multicolumn{1}{c}{$C V_{0}(T, X)$} \\
\hline$T$ completely regular Hausdorff $k_{\mathbf{R}}$ & $\begin{array}{l}C T, X)_{\text {co }} \text { : continuous functions, } \\
\text { with the compact-open topology }\end{array}$ \\
$V=\left\{\lambda \chi_{K} \mid \lambda \geq 0, K\right.$ compact in $\left.T\right\}$ & \\
\hline $\begin{array}{l}S \text { locally compact Hausdorff } \\
X \text { quasi-complete locally convex }\end{array}$ & $\begin{array}{l}C_{b}(S, X)_{\beta}: \text { bounded continuous } \\
\text { functions, with the strict to- } \\
\text { pology }\end{array}$ \\
\hline $\begin{array}{l}S \text { locally compact Hausdorff } \\
X \text { Banach }\end{array}$ & $\begin{array}{l}C_{0}(S, X): \text { continuous functions } \\
\text { vanishing at infinity, with }\end{array}$ \\
$V=C_{b}^{+}(S)$ & sup-norm \\
\hline$S$ locally compact Hausdorff, & $\begin{array}{l}C_{c}(S, X)_{i}: \text { continuous functions } \\
\text { with compact support, with the }\end{array}$ \\
countable at infinity & usual inductive limit topology \\
$X$ Banach & \\
$V=C^{+}(S)$ &
\end{tabular}

Facts (cf. $[6,7,8])$. (a) $C V_{0}(T, X)$ is complete whenever $X$ is complete and $T$ is a $V_{\mathbf{R}}$-space, i.e. a function $f: T \rightarrow \mathbf{R}$ is continuous as soon as its restrictions $f \mid\{t \in T \mid v(t) \geq 1\}$ are continuous for all $v \in V$.

(b) If $T$ is a $V_{\mathbf{R}}$-space and $X$ is quasi-complete, then we have:

$$
\begin{gathered}
C V_{0}(T, X) \cong L_{e}\left(X_{c}^{\prime}, C V_{0}(T)\right) \\
F \mapsto F^{*}:\left\{x^{\prime} \mapsto x^{\prime} \circ F\right\} .
\end{gathered}
$$

In [9], Bogdanowicz showed that a bounded sequence $\left(F_{n}\right)_{n}$ in $C(K, X), K$ compact Hausdorff, $X$ Banach, converges weakly to $F \in$ $C(K, X)$ if and only if $\left(F_{n}(t)\right)_{n}$ converges weakly (in $\left.X\right)$ to $F(t)$ for all $t \in T$. We now use the ideas of $\$ 2$ to show that the corresponding result holds for just any of the weighted spaces $C V_{0}(T, X)$. 
4.2 THEOREM. Let $T$ be a completely regular Hausdorff space, and $X a$ quasi-complete locally convex space. Let $V>0$ be a directed family of weights on $T$ such that $T$ is a $V_{\mathbf{R}}$-space and that for every $t \in T$ there exists $f \in C V_{0}(T)$ with $f(t) \neq 0$.

Then a sequence $\left(F_{n}\right)_{n} \subset C V_{0}(T, X)$ converges weakly to $F \in$ $C V_{0}(T, X)$ if and only if it is bounded and $\left(F_{n}(t), x^{\prime}\right)_{n}$ converges to $\left(F(t), x^{\prime}\right)$ for all $t \in T$ and all $x^{\prime} \in \operatorname{ext} U^{0}, U \in \mathcal{Q}_{X}$.

This follows from a combination of Theorem 2.4, fact (b) above, and results of Summers [58, Thm. 4.6] and Goullet de Rugy [26, Cor. 4.5] (see also [50]) which state that under the assumptions on $T$ and $V, \operatorname{ext}\left(B_{v}\right)_{+}^{0}=$ $\left\{v(t) \delta_{t} \mid t \in N(v)\right\} \cup\{0\}$, where $B_{v}=\left\{f \in C V_{0}(T)|v(t)| f(t) \mid \leq 1\right.$ for all $t \in T\}$, and $N(v)=\{t \in T \mid v(t)>0\}$.

Clearly, also Theorem 2.6 and the results of $\$ 1$ allow pointwise-weak versions for weak compactness properties of subsets of $C V_{0}(T, X)$. These consequences are left to the interested reader. We only note here that Proposition 2.7 for the space $C(T, X)_{\mathrm{co}} \cong L_{e}\left(X_{c}^{\prime}, C(T)_{\mathrm{co}}\right) \cong$ $L_{e}\left(\left(C(T)_{\mathrm{co}}\right)_{c}^{\prime}, X\right)$ specializes to Grothendieck's classical result [28, II. Lemma 1] on weak compactness in this function space.

In view of the discussion preceding Theorem 4.2, Proposition 2.7 allows us to derive the following general result:

4.3 TheOREM. Let $E$ denote any of the function spaces $C(T, X)_{\mathrm{co}}$, $C_{b}(S, X)_{\beta}, C_{0}(S, X)$, or $C_{c}(S, X)_{i}, S, T$ and $X$ as specified in Examples 4.1. Then a subset $H$ of $E$ is weakly relatively compact in $E$ if and only if

(i) $H$ is bounded in $E$,

(ii) $H(t)$ is weakly relatively compact in $X$ for all $t \in T$ (resp. $t \in S$ ), and

(iii) every pointwise-weak limit $F: T \rightarrow X($ resp. $F: S \rightarrow X)$ of a net in $H$ belongs to $E$.

(From results in [50] it will follow that Theorem 4.3 holds for any space $C V_{0}(T, X)$ as specified in Theorem 4.2.)

Note that our results on weak convergence and weak compactness in continuous function spaces contain corresponding results of [37, Cor. 3.7] and $[\mathbf{6 1}]$ as particular cases.

In concluding this section on weighted function spaces, we want to point out that the results considered so far also apply to weighted spaces of holomorphic vector-valued functions (see [4, 2.9]), and to spaces of holomorphic vector-valued functions in infinitely many variables. In this 
very last context, note the following result of Aron/Schottenloher [3]: Given $X$ and $Y$ (complex) locally convex spaces, $Y$ quasi-complete, and $U$ an open subset of $X$, the space $H(U, Y)_{\text {co }}$ is a topological linear subspace of

$$
\begin{aligned}
& L_{e}\left(Y_{c}^{\prime}, H(U)_{\mathrm{co}}\right): H(U, Y)_{\mathrm{co}} \underset{\hookrightarrow}{\sim} L_{e}\left(Y_{c}^{\prime}, H(U)_{\mathrm{co}}\right) \\
& F \mapsto F^{*}:\left\{y^{\prime} \mapsto y^{\prime} \circ F\right\} .
\end{aligned}
$$

Applications of our results to this function space are left to the interested reader.

As a final example, we now investigate Pettis integrable functions.

Let $(\Omega, \Sigma, \mu)$ be a finite measure space.

Definition (cf. [59]). Let $X$ be a quasi-complete locally convex space.

(a) A function $F: \Omega \rightarrow X$ is said to be Pettis integrable if

(i) $x^{\prime} \circ F$ is measurable for all $x^{\prime} \in X^{\prime}$,

(ii) $x^{\prime} \circ F \in L^{1}(\mu)$ for all $x^{\prime} \in X^{\prime}$, and

(iii) for $E \in \Sigma$ there exists an element $\int_{E} F d \mu \in X$ such that $\left(\int_{E} F d \mu, x^{\prime}\right)=\int_{E}\left(x^{\prime} \circ F\right) d \mu$ for all $x^{\prime} \in X^{\prime}$.

(b) A function $F: \Omega \rightarrow X$ is said to be measurable if there exists a sequence $\left(F_{n}\right)_{n}$ of $\mu$-simple functions which converge $\mu$-a.e. to $F$.

(c) The Pettis topology on the space $P(\mu, X)$ of all Pettis integrable functions $F: \Omega \rightarrow X$ is generated by the seminorms

$$
p_{U}(F)=\sup \left\{\int\left|x^{\prime} \circ F\right| d \mu \mid x^{\prime} \in U^{0}\right\}, \quad U \in \mathcal{Q}_{X} \text {. }
$$

4.4 Lemma. Whenever $F: \Omega \rightarrow X$ is Pettis integrable, $X$ quasi-complete, then the associated linear operator $F^{*}: X^{\prime} \rightarrow L^{1}(\mu), F^{*}\left(x^{\prime}\right)=x^{\prime} \circ F$, is weak*-weakly continuous.

Proof. The linear operator $T_{F}: L^{\infty}(\mu) \rightarrow X, T_{F}(g)=\int g F d \mu$, is well defined and weak*-weakly continuous, cf. I, II, and III on pages 64/65 of [59]. It is easy to see that $F^{*}=\left(T_{F}\right)^{\prime}$.

According to Lemma 4.4, whenever we want to apply our operatortheoretic results to spaces of Pettis integrable functions, we have to specify conditions on $F, \Omega$ and $X$ which ensure that the operators $F^{*}$ actually are $c$-continuous, i.e. that they transform equicontinuous subsets of $X^{\prime}$ into relatively compact subsets of $L^{1}(\mu)$. 
4.5 Proposition. Let $X$ be a quasi-complete locally convex space, $F$ : $\Omega \rightarrow X$ Pettis integrable, and consider the associated linear operator $F^{*}$ : $X^{\prime} \rightarrow L^{1}(\mu), F^{*}\left(x^{\prime}\right)=x^{\prime} \circ F$. If

(a) $F$ is measurable, in particular, if $X$ is separable, or if

(b) $(\Omega, \Sigma, \mu)$ is a perfect measure space [51], and $F$ is bounded, then $F^{*} \in L\left(X_{c}^{\prime}, L^{1}(\mu)\right)$.

Proof. The case when $X$ is Banach is well known: for $F$ measurable, see [24]; if $X$ is separable, then $F$ is measurable, according to Pettis' measurability criterion; and the compactness of $F^{*}$ for $X$ Banach under assumption (c) is a result of C. Stegall, cf. [24] and [38]. We now reduce the general case to the Banach space case. Let $\mathcal{Q}_{X}$ be a zero neighbourhood base in $X$, all $U \in \mathcal{Q}_{X}$ closed convex and circled, and denote by $X_{U}$ the usual Banach space associated with $U \in \mathcal{Q}_{X}: X_{U}=$ completion of $\left(X / N(U), q_{U}\right)$, where $q_{U}$ is the Minkowski-functional of $U$, and $N(U)=$ $\left\{x \in X \mid q_{U}(x)=0\right\}$. Moreover, denote by $j_{U}$ the canonical projection $j_{U}$ : $X \rightarrow X_{U}$, and recall that $\left(j_{U}\right)^{\prime}:\left(X_{U}\right)^{\prime} \rightarrow X^{\prime}$ is an isometry onto $X_{U^{0}}^{\prime}=$ the span of $U^{0}$ in $X^{\prime}$, with the norm unit ball $U^{0}$.

Given $F: \Omega \rightarrow X$ Pettis integrable, consider the maps

$$
F_{U}=j_{U} \circ F: \Omega \stackrel{F}{\rightarrow} X \stackrel{j_{U}}{\rightarrow} X_{U}, \quad U \in \mathcal{Q}_{X} .
$$

It is easy to see that all $F_{U}$ are Pettis integrable as well, that they are strongly measurable, or bounded, whenever $F$ is, and that they are strongly measurable whenever $X$ is separable, for then all $X_{U}$ are separable. Hence, according to the first part of the proof, all maps $\left(F_{U}\right)^{*}$ : $\left(X_{U}\right)^{\prime} \rightarrow L^{1}(\mu)$ are compact. But we have the identities:

$$
\begin{aligned}
\left(F_{U}\right)^{*}\left(B\left(\left(X_{U}\right)^{\prime}\right)\right) & =B\left(\left(X_{U}\right)^{\prime}\right) \circ j_{U} \circ F \\
& =\left(j_{U}\right)^{\prime}\left(B\left(\left(X_{U}\right)^{\prime}\right)\right) \circ F=U^{0} \circ F=F^{*}\left(U^{0}\right),
\end{aligned}
$$

and this completes the proof.

We can now combine Proposition 4.5 with the results of $\$ 2$.

4.6 TheOREM. Let $(\Omega, \Sigma, \mu)$ be a finite measure space, $X$ a quasi-complete locally convex space, and $F,\left(F_{n}\right)_{n} \subset P(\mu, X)$ Pettis integrable functions $\Omega \rightarrow X$. Moreover, suppose that for $(\Omega, \Sigma, \mu), X$ and $\left\{F,\left(F_{n}\right)_{n}\right\}$ any of the assumptions (a) or (b) of Proposition 4.5 is fulfilled. Then the sequence $\left(F_{n}\right)_{n}$ converges weakly (with respect to the Pettis topology on $P(\mu, X)$ ) to $F$ if and only if it is bounded and $\left(\int_{E} x^{\prime} \circ F_{n} d \mu\right)_{n}$ converges to $\int_{E} x^{\prime} \circ F d \mu$ for all $E \in \Sigma$ and all $x^{\prime} \in \operatorname{ext} U^{0}, U \in \mathcal{Q}_{X}$. 
As before, also the other results of $\S \S 1$ and 2 directly translate into weak compactness criteria for sets of Pettis integrable functions. Let us note just on more special case. Others are left to the interested reader.

Taking into account the special weak convergence criteria in an $\mathrm{L}^{1}(\mu)$-space, Proposition 1.6 translates into the following result:

4.7 Theorem. Let $(\Omega, \Sigma, \mu)$ be a finite measure space, and $X$ a Fréchet space. Denote by $P_{1}(\mu, X)$ the space of all (strongly) measurable Pettis integrable functions with the Pettis topology (note that $P_{1}(\mu, X)=P(\mu, X)$ whenever $X$ is separable $)$. Then a subset $H$ of $P_{1}(\mu, X)$ is weakly conditionally compact if and only if

(i) the set $x^{\prime} \circ H \subset L^{1}(\mu)$ is bounded and uniformly integrable for all $x^{\prime} \in X^{\prime}$, and

(ii) the set $\left\{\int_{E} F d \mu \mid F \in H\right\}$ is weakly conditionally compact in $X$ for all $E \in \Sigma$.

Theorems 4.6 and 4.7 extend corresponding results of D. R. Lewis [37, Cors. 3.4, 3.5].

\section{REFERENCES}

1. N. I. Achieser and I. M. Glasmann, Theorie der linearen Operatoren im Hilbert-Raum, Akademie-Verlag, Berlin 1968.

2. D. Amir and J. Lindenstrauss, The structure of weakly compact sets in Banach spaces, Ann. of Math. 88 (1968), 35-46.

3. R. M. Aron and M. Schottenloher, Compact holomorphic mappings on Banach spaces and the approximation property, J. Functional Analysis, 21 (1976), 7-30.

4. K.-D. Bierstedt, Gewichtete Räume stetiger vektorwertiger Funktionen und das injektive Tensorprodukt I, J. Reine Angew. Math., 259 (1973), 186-210.

5. __ Gewichtete Räume stetiger vektorwertiger Funktionen und das injektive Tensorprodukt II, J. Reine Angew. Math., 260 (1973), 133-146.

6 . The approximation property for weighted function spaces, Bonner Math. Schriften, 81 (1975), 3-25.

7. __ Tensor products of weighted spaces, Bonner Math. Schriften, 81 (1975), 26-58.

8. The approximation-theoretic localization of Schwartz's approximation property for weighted locally convex function spaces and some examples, Preprint 1979.

9. W. M. Bogdanowicz, Representation of linear continuous functionals on the space $C(X, Y)$ of continuous functions from compact $X$ into locally convex $Y$, Proc. Japan Acad., 42 (1967), $1122-1127$.

10. J. Bourgain and M. Talagrand, Compacité extrémale, Proc. Amer. Math. Soc., 80 (1980), 68-72.

11. J. K. Brooks and P. W. Lewis, Operators on continuous function spaces and convergence in the space of operators, Advances in Math., 29 (1978), 157-177.

12. R. C. Buck, Operator algebras and dual spaces, Proc. Amer. Math. Soc., 3 (1952), 681-687.

13. Bounded continuous functions on a locally compact space, Michigan Math. J., 5 (1958), 95-104. 
14. R. C. Busby, Double centralizers and extensions of $C^{*}$-algebras, Trans. Amer. Math. Soc., 132 (1968), 79-99.

15. H. S. Collins, Strict, weighted, and mixed topologies and applications, Advances in Math., 19 (1976), 207-237.

16. H. S. Collins and W. Ruess, Duals of spaces of compact operators and vector-valued functions, To appear in Studia Math.

17. , Duality for spaces of compact operators, Preprint.

18. M. De Wilde, Pointwise compactness in spaces of functions and R. C. James' Theorem, Math. Ann., 208 (1974), 33-47.

19. J. Diestel and J. J. Uhl, Jr., Vector measures, Amer. Math. Soc. Math. Surveys, 15 (1977).

20. H. Fakhouri, Approximation par des opérateurs compacts ou faiblement compacts à valeurs dans $C(X)$, Ann. Inst. Fourier, 27 (1977), 147-167.

21. H. Fakhouri, Quelques propriétés de l'espace des opérateurs, Studia Math., 64 (1979), 239-247.

22. K. Floret, Lectures on Weakly Compact Sets, Lecture Notes in Math., Vol. 801, Springer, Berlin, Heidelberg, New York 1980.

23. D. H. Fremlin, D. J. H Garling and R. G. Haydon, Bounded measures on topological spaces, Proc. London Math. Soc. III, Ser. 25, (1972), 115-136.

24. D. H. Fremlin and M. Talagrand, A decomposition theorem for additive set-functions, with applications to Pettis integrals and erogdic means, Math. Z. 168 (1979), 117-142.

25. J. Gil de Lamadrid, Measures and Tensors II, Canad. J. Math., 18 (1966), 762-793.

26. A. Goullet de Rugy, Espaces de fonctions ponderables, Israel J. Math., 12 (1972), $147-160$.

27. A. Grothendieck, Critères de compacité dans les espaces fonctionnels généraux, Amer. J. Math., 74 (1952), 168-186.

28. __ Sur certains espaces de fonctions holomorphes I, II, J. Reine Angew. Math., 192 (1953), 35-64, 77-95.

29. __ Espaces vectoriels topologiques, Sociedade Mat. S. Paulo, Sao Paulo, 1954.

30. __ Produits tensoriels topologiques et espaces nucléaires, Memoirs Amer. Math. Soc. $16(1955)$.

31. S. Heinrich, On the reflexivity of the Banach space $L(E, F)$, Functional Analysis Appl., 8 (1974), 186-187. Translated from Funktsionalnyi Analiz Prilozheniya 8 (1974).

32. J. R. Holub, Reflexivity of $L(E, F)$, Proc. Amer. Math. Soc., 39 (1973), 175-177.

33. J. Horváth, Topological Vector Spaces and Distributions I, Addison-Wesley, Reading 1966.

34. H. D. Junghenn, Tensor products and almost periodicity, Proc. Amer. Math. Soc., 43 (1974), 99-105.

35. N. J. Kalton, Spaces of compact operators, Math. Ann. 208 (1974), 267-278.

36. G. Köthe, Topological Vector Spaces I, Springer, Berlin, Heidelberg, New York 1969.

37. D. R. Lewis, Conditional weak compactness in certain inductive tensor products, Math. Ann., 201 (1973), 201-209.

38. F. Lust, Produits tensoriels injectifs d'espaces faiblement séquentiellement complets, Coll. Math., 33 (1975), 289-290.

39. L. Nachbin, Elements of Approximation Theory, Van Nostrand Math. Studies, 14 (1967).

40. K. Noureddine, Espaces du type $D_{b}$, C. R. Acad. Sci. Paris, 276 (1973), A1301-1303.

41. H. R. Pitt, A note on bilinear forms, J. London Math. Soc. II, (1936), 174-180.

42. J. B. Prolla, Weighted spaces of vector-valued continuous functions, Ann. Mat. Pura Appl., 89 (1971), 145-158.

43. J. Rainwater, Weak convergence of bounded sequences, Proc. Amer. Math. Soc., 14 (1963), 999. 
44. H. P. Rosenthal, $A$ characterization of Banach spaces containing $l^{1}$, Proc. Nat. Acad. Sci. USA 71 (1974), 2411-2413.

45. W. H. Ruckle, Reflexivity of $L(E, F)$, Proc. Amer. Math. Soc., 34 (1972), 171-174.

46. W. Ruess, On the locally convex structure of strict topologies, Math. Z., 153 (1977), 179-192.

47. The strict topology and DF spaces, Proc. Conf. Functional Analysis, Paderborn 1976. North Holland Math. Studies 27 (1977), 105-118.

48. _ [Weakly] Compact operators and DF spaces, Pacific J. Math., 98 (1982), 419-441.

49. W. Ruess and C. Stegall, Extreme points in duals of operator spaces, to appear in Math. Ann.

50. W. Ruess and W. H. Summers, Compactness and weak compactness in spaces of vector-valued continuous functions, In preparation.

51. V. V. Sazonov, On perfect measures, Amer. Math. Soc. Translations, (2) 48 (1965), 224-254.

52. R. Schatten, The space of completely continuous operators on a Hilbert space, Math. Ann., 134 (1957), 47-49.

53. L. Schwartz, Espaces de fonctions différentiables à valeurs vectorielles, J. d'Analyse Math., 4 (1954-55), 88-148.

54. __ Théorie des distributions à valeurs vectorielles I, Ann. Inst. Fourier, 7 (1957), $1-139$.

55. F. D. Sentilles, Bounded continuous functions on a completely regular space, Trans. Amer. Math. Soc., 168, (1972), 311-336.

56. F. D. Sentilles and D. C. Taylor, Factorization in Banach algebras and the general strict topology, Trans. Amer. Math. Soc., 142 (1969), 141-152.

57. C. Stegall, The Radon-Nikodym property in conjugate Banach spaces, Trans. Amer. Math. Soc., 206 (1975), 213-223.

58. W. H. Summers, A representation theorem for biequicontinuous completed tensor products of weighted spaces, Trans. Amer. Math. Soc., 146 (1969), 121-131.

59. G. E. F. Thomas, Integration of functions with values in locally convex Suslin spaces, Trans. Amer. Math. Soc., 212 (1975), 61-81.

60. L. N. Tsitsas, On weak sequential completeness in biprojective tensor product spaces, Proc. Amer. Math. Soc., 60 (1976), 119-123.

61. On weak compactness in biprojective tensor product spaces, Math. Nachr., 78 (1977), 297-308.

62. $\ldots$ On the heredity of weak compactness in biprojective tensor product spaces, Studia Math., 61 (1977), 1-6.

63. __ On the semi-reflexivity of biprojective tensor product spaces, J. Analysis Appl.,. 69 (1979), 505-510.

Received September 23, 1981. Research performed while the second named author was a visitor in the Department of Mathematics at Louisiana State University, Baton Rouge.

Louisiana State UNIVERSITY

BATON ROUGE, LA 70803 



\section{PACIFIC JOURNAL OF MATHEMATICS EDITORS}

DONALD BABBITT (Managing Editor)

University of California

Los Angeles, CA 90024

Hugo Rossi

University of Utah

Salt Lake City, UT 84112

C. C. Moore and Arthur Ogus

University of California

Berkeley, CA 94720
J. DugunduI

Department of Mathematics

University of Southern California

Los Angeles, CA 90089-1113

R. FinN and H. SAmelson

Stanford University

Stanford, CA 94305

\section{ASSOCIATE EDITORS}
R. ARENS
E. F. BECKENBACH
B. H. NeUmaNN
F. WolF
K. YoshidA (1906-1982)

\section{SUPPORTING INSTITUTIONS}

UNIVERSITY OF ARIZONA

UNIVERSITY OF BRITISH COLUMBIA

CALIFORNIA INSTITUTE OF TECHNOLOGY

UNIVERSITY OF CALIFORNIA

MONTANA STATE UNIVERSITY

UNIVERSITY OF NEVADA, RENO

NEW MEXICO STATE UNIVERSITY

OREGON STATE UNIVERSITY
UNIVERSITY OF OREGON

UNIVERSITY OF SOUTHERN CALIFORNIA

STANFORD UNIVERSITY

UNIVERSITY OF HAWAII

UNIVERSITY OF TOKYO

UNIVERSITY OF UTAH

WASHINGTON STATE UNIVERSITY

UNIVERSITY OF WASHINGTON 


\section{Pacific Journal of Mathematics}

\section{Vol. 106, No. $1 \quad$ November, 1983}

John Ballard, Clifford's theorem for algebraic groups and Lie algebras . . . . . 1

Philip Richard Bartick, II and Edwin Duda, Finite-to-one open mappings

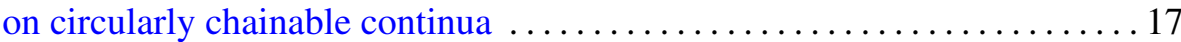

Frank Hayne Beatrous, Jr., $H^{\infty}$-interpolation from a subset of the

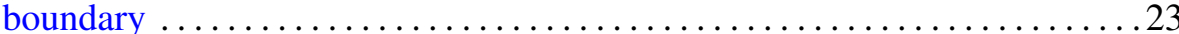

Sterling K. Berberian, Expectations in semifinite algebras ............ 33

Heron S. Collins and Wolfgang Ruess, Weak compactness in spaces of compact operators and of vector-valued functions $\ldots \ldots \ldots \ldots \ldots \ldots . \ldots 45$

David Downing and William O. Ray, Renorming and the theory of

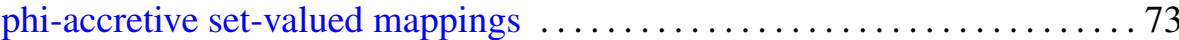

Harvey Charles Greenwald, On the theory of homogeneous Lipschitz

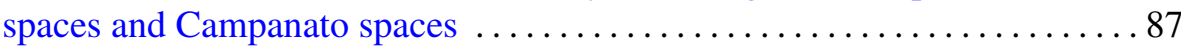

Irwen Valle Guadalupe and Lucio Ladislao Rodriguez, Normal curvature

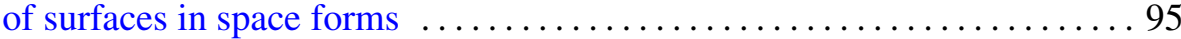

Boju Jiang, On the computations of the Nielsen number ............... 105

William H. Julian, Ray Mines, III and Fred Richman, Alexander

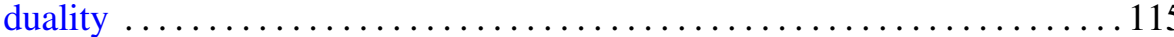

Midori Kobayashi, The connected component of the idèle class group of an

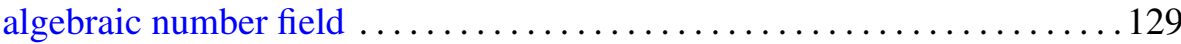

Ib Henning Madsen, Charles B. Thomas and C. Terence C. (Charles)

(Clegg) Wall, Topological spherical space form problem. III.

Dimensional bounds and smoothing

A. Sapounakis, The existence of strong liftings for totally ordered measure

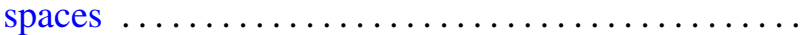

Gary Seitz, The root subgroups for maximal tori in finite groups of Lie type

Laurent Siebenmann and James M. Van Buskirk, Construction of irreducible homology 3-spheres with orientation reversing involution 